\title{
Systematic Review \\ Factors Contributing to Medication Adherence in Patients with a Chronic Condition: A Scoping Review of Qualitative Research
}

\author{
Kirsi Kvarnström ${ }^{1,2, *(\mathbb{D}}$, Aleksi Westerholm ${ }^{2}\left(\mathbb{D}\right.$, Marja Airaksinen ${ }^{2}\left(\mathbb{D}\right.$ and Helena Liira ${ }^{3,4}$ \\ 1 HUS Pharmacy, University of Helsinki and Helsinki University Hospital, 00290 Helsinki, Finland \\ 2 Clinical Pharmacy Group, Division of Pharmacology and Pharmacotherapy, Faculty of Pharmacy, \\ University of Helsinki, 00014 Helsinki, Finland; aleksi.westerholm@helsinki.fi (A.W.); \\ marjaairaksinen@gmail.com (M.A.) \\ 3 Department of General Practice, University of Helsinki, 00290 Helsinki, Finland; helena.liira@helsinki.fi \\ 4 Unit of Primary Health Care, University of Helsinki and Helsinki University Hospital, \\ 00290 Helsinki, Finland \\ * Correspondence: kirsi.kvarnstrom@hus.fi
}

\section{check for}

updates

Citation: Kvarnström, K.;

Westerholm, A.; Airaksinen, M.; Liira,

H. Factors Contributing to

Medication Adherence in Patients

with a Chronic Condition: A Scoping

Review of Qualitative Research.

Pharmaceutics 2021, 13, 1100.

https://doi.org/10.3390/

pharmaceutics 13071100

Academic Editors:

Przemysław Kardas and Jaehwi Lee

Received: 28 May 2021

Accepted: 15 July 2021

Published: 20 July 2021

Publisher's Note: MDPI stays neutral with regard to jurisdictional claims in published maps and institutional affiliations.

Copyright: (c) 2021 by the authors. Licensee MDPI, Basel, Switzerland. This article is an open access article distributed under the terms and conditions of the Creative Commons Attribution (CC BY) license (https:// creativecommons.org/licenses/by/ $4.0 /)$.

\begin{abstract}
Introduction: Medication adherence continues to be a significant challenge in healthcare, and there is a shortage of effective interventions in this area. This scoping review studied the patient-related factors of medication adherence. Methods: We searched Medline Ovid, Scopus, and Cochrane Library from January 2009 to June 2021 to find the most recent original qualitative studies or systematic reviews that addressed the patient-related factors of medication adherence in treating chronic conditions. We used the PRISMA-ScR checklist to ensure the quality of the study. Results: The initial search revealed 4404 studies, of which we included 89 qualitative studies in the scoping review. We inductively organized the patient-related factors causing barriers, as well as the facilitators to medication adherence. The studies more often dealt with barriers than facilitators. We classified the factors as patient-specific, illness-specific, medication-related, healthcare and system-related, sociocultural, as well as logistical and financial factors. Information and knowledge of diseases and their treatment, communication, trust in patient-provider relationships, support, and adequate resources appeared to be the critical facilitators in medication adherence from the patient perspective. Discussion and conclusions: Patients are willing to discuss their concerns about medications. Better communication and better information on medicines appear to be among the critical factors for patients. The findings of this scoping review may help those who plan further interventions to improve medication adherence.
\end{abstract}

Keywords: medication adherence; patient compliance; primary non-adherence; patient-related factors; qualitative research; barriers; facilitators; scoping review; chronic conditions

\section{Introduction}

Medication adherence continues to be a significant challenge in healthcare, and there is a shortage of effective interventions. In 2003, the World Health Organization identified that only $50 \%$ of chronically ill patients take their medication as prescribed in developed countries [1]. Although there is a wealth of controlled trials on interventions to improve adherence, current methods of improving medication adherence for chronic health problems are mostly complex and not effective [2-4]. Previous studies and systematic reviews have combined the existing evidence of adherence interventions [3]. Nevertheless, it seems that there is still a lack of understanding about the complexity of medication adherence from the patient's perspective.

Medication nonadherence is associated with poorer health outcomes [5]. If patients do not gain the expected health benefits from their medication because of nonadherence, the burden of health care costs increases for both patients and society in general [6-8]. The same factors that improve medication adherence may also decrease it [9-11]. The patient can 
experience medication-related burdens, which may negatively affect adherence [12]. On the other hand, a patient's nonadherence can be seen as a behavioural problem related to their course of action [13]. Many studies have focused on medication adherence related to some specific illness instead of medication adherence in general. The studies may be lacking the input of patients, while the viewpoint of healthcare professionals may have been dominant. Patients struggle in reconciling daily life with comorbidity and multiple medications may be poorly understood [14]. Patient-centred care requires a greater understanding of the daily decisions patients need to make in order to manage a complex medication regimen.

Many theories have been applied to explain medication adherence behaviour. The information-motivation-behavioural skills (IMB) model is a widely used social behaviour model to explain medication adherence among chronically ill patients $[15,16]$. According to the model, the following three dimensions influence adherence behaviour: (1) Information and knowledge about the need for essential behaviour, (2) Motivation to make necessary behavioural changes and (3) The required behavioural skills to achieve the desired behaviour.

The model may explain patients' actions regarding their rational use of medicines. Patients may not have sufficient information and understanding about their illness or medication to make an adequate decision, and they can seek the information from various sources [17]. Patient motivation is crucial to cope with multiple medications and make these fit into daily life. On the other hand, there may be personal reasons and system and organisation-specific barriers, which can lead to unwanted behaviour and medication nonadherence [18]. However, no theory alone seems to explain a patient's adherence to medication because there may be external factors that can also affect adherence.

We need a more patient-focused approach to medication adherence and a better understanding of this complex phenomenon. This scoping review aimed at a better understanding of patients' views on medication adherence and analysing the contributing factors as to why patients are not taking the medication as prescribed in outpatient settings. We wanted to understand this complex phenomenon in depth and summarize our findings in this scoping review.

\section{Materials and Methods}

We used the PRISMA-ScR checklist to ensure the quality of the study. The present scoping review is reported based on the guidelines of Preferred Reporting Items for Systematic reviews and Meta-Analyses extension for Scoping Reviews [19]. The PRISMA-ScR is available from the authors upon request.

\subsection{Search Strategy}

The literature search for eligible qualitative studies was conducted on 23 September 2019, using MEDLINE (Ovid), Scopus, and the Cochrane Library, with the assistance of an information specialist at the Helsinki University Library. The search was updated on 9 June 2021. We included articles that were published from January 2009 to June 2021. We wanted to focus on the most recent publications, so we did not include the publications published before the year 2009. We limited the article search to English language studies and articles published in peer-reviewed journals. We used the following wide range of search terms related to medication, drug, medicine, adherence, non-adherence, compliance, non-compliance, patient, experience, fear, beliefs, knowledge, attitudes, behaviour, communication, reason, and cause. Relating to the study design, our search terms were: qualitative, interview, focus group, questionnaire, observation, study, and research. An example of the search strategy is presented in the included appendix material (Appendix A).

\subsection{Inclusion and Exclusion Criteria}

We were interested in the phenomena leading to medication adherence and nonadherence from the patient perspective. Therefore, we included qualitative studies where 
the primary focus was understanding the complexity of medication adherence described by patients who were being treated for chronic conditions. We included original qualitative studies and systematic reviews if the study population consisted of people of 18 years or older and patients with at least one chronic condition. We also required that the primary focus was on patients' experiences and attitudes towards medication adherence. We did not require comparison groups. It was mandatory that the researchers had used qualitative methods both for data collection and data analysis. We wanted to study the phenomena in general, so we excluded studies where the primary study population consisted of children or adolescents under 18 years or patients with an acute illness who were pregnant or drug or alcohol users. We also excluded conference papers, quantitative methods and mixed methods studies, as well as studies that collected data using qualitative methods, but data was analysed using quantitative methods.

\subsection{Study Selection}

The systematic searches for eligible articles retrieved 4404 studies. After duplicates were removed, the researchers (KK, AW, HL) independently screened the titles and abstracts for eligibility using the online software, Covidence. If one or two reviewers identified the article as relevant, we carried out a full-text review. We solved any disagreements via discussions and reaching a consensus. After the title and abstract screening, two reviewers $(\mathrm{KK}, \mathrm{AW})$ independently screened the full text of selected articles. Disagreements were resolved through discussions with the third reviewer (HL) for final inclusion. The articles were selected in several parts, which allowed the reviewers to have a regular discussion of the eligibility criteria, ensuring the same understanding of the criteria, and the criteria remaining the same throughout the article selection phase.

We did not assess the risk of bias of the included studies. As in many scoping reviews, the goal was to describe the phenomena surrounding patients and medication adherence [20].

\subsection{Data Extraction}

We constructed a template to carry out the data extraction using the Covidence online platform. Two reviewers (KK, HL) independently extracted the data, and the results were reviewed and verified by both reviewers for quality and clarity. We resolved the discrepancies by discussions and reaching a consensus. The data extraction template first focused on the study design, illness, context and concept of the studies, as well as barriers and facilitators to medication adherence. After extracting a third of the studies, we constructed a more specific classification for barriers and facilitators to medication adherence and re-extracted the material from the beginning with the wider list of items. We elaborated this classification further during the analysis of the results. We noted patients knowledge of their illness and its treatment. Motivation and behaviour skills seemed to be essential and correlated to good medication self-management during the analysis. Therefore, we decided to apply the IMB model as part of the classification of facilitators to medication adherence $[15,16]$. The authors provide by request the final list of data items documented in the Covidence extraction form.

\section{Results}

We included 89 original peer-reviewed articles in this scoping review (Figure 1). The study design in all the articles was qualitative and carried out in community or outpatient settings (Table A1). The studies were conducted in 36 different countries: The United States $(n=19)$, The United Kingdom $(n=10)$, South Africa $(n=4)$, Australia $(n=3)$, Canada $(n=3)$, Malaysia $(n=3)$, The Netherlands $(n=2)$, Sweden $(n=2)$, Indonesia $(n=2)$, Iran $(n=2)$ and one study from each of the following countries: Belgium, Norway, Portugal, Spain, Switzerland, Germany, Ireland, France, Italy, Singapore, New Zealand, Taiwan, Jordan, Pakistan, Kuwait, Saudi-Arabia, Vietnam, Uganda, Tanzania, Kenya, Eswatini, 
Ethiopia, Namibia and Lesotho. There was one study where both Nepal and Australia were involved and one study where Italy, Portugal and Poland were involved.

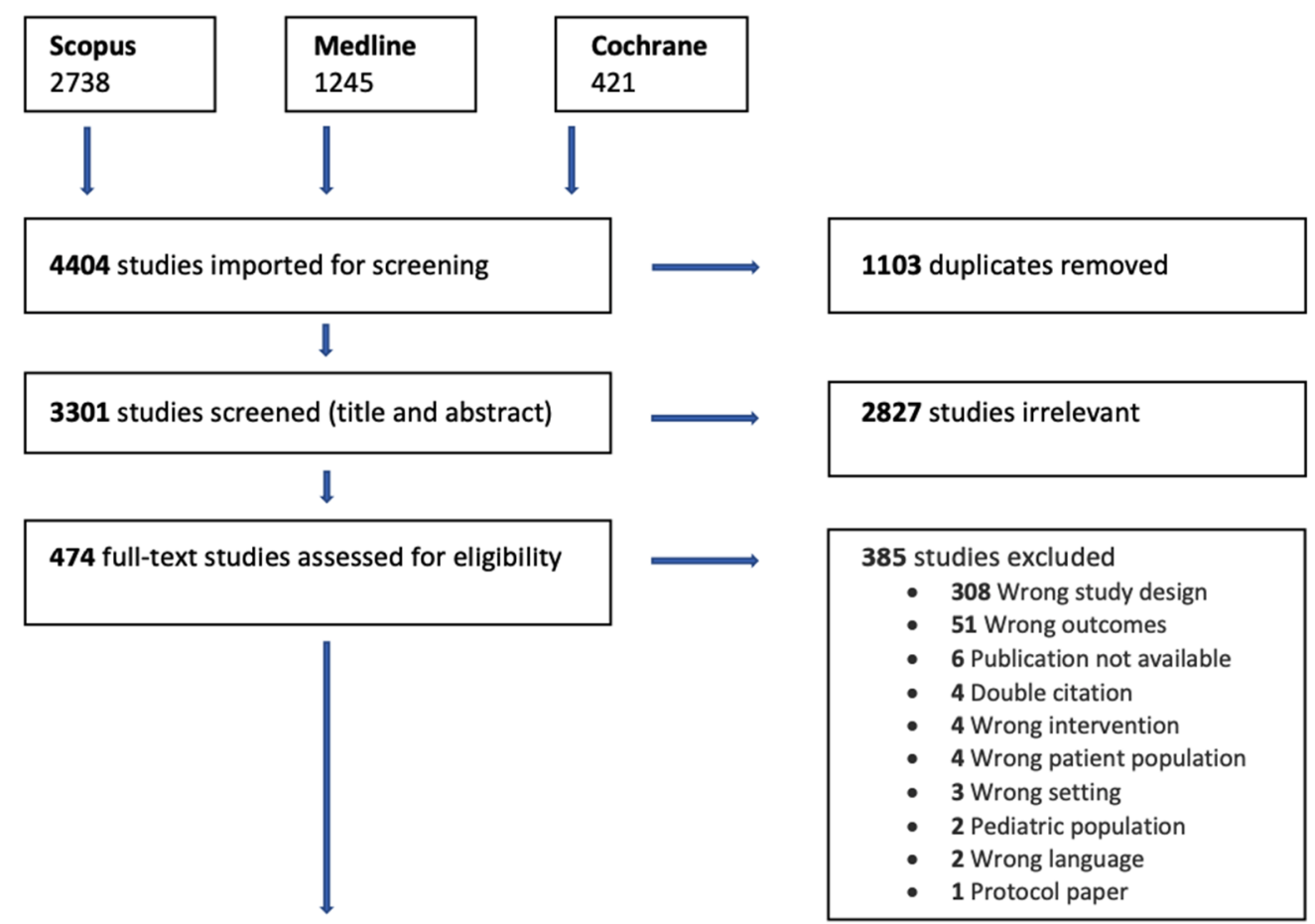

89 studies included

Figure 1. PRISMA flow diagram of the study selection process.

Our review covered 13 systematic reviews on medication adherence (Table A2). Seven of them focused on patients with cardiovascular disease or type two diabetes [21-27], one on patients with rheumatoid arthritis [28], one on patients with breast cancer [29], two on patients with chronic kidney disease or kidney transplants $[10,30]$ and two with no specific illness [31,32].

There were 17 studies that had a behaviour theory-based approach to medication adherence (Table 1). The theories that appeared were: Andersen's Behavioural Model [33,34], Roy Adaptation Model [35], Common-Sense Model of Self-Regulation [36], Social-Ecological Model [37,38], Therapeutic Alliance [39], Dowell's Therapeutic Alliance Model and Leventhal's Common Sense Model [40], Health Literacy Pathway Model [41], ABC Taxonomy and Three-Factor Model [32], Health Belief Model [42-45], Naturalistic Decision Model [46] and Stages of Change Model [47]. One of the studies did not have a theory-based approach in the beginning, but many of the findings fitted together with the Information-MotivationBehaviour model [48].

The context of most of the studies was an outpatient setting, either in primary or secondary care (Table A1). The studies' concept varied from the rationale of taking medication to understanding patients' beliefs, practices, and reasons for nonadherence. 
Table 1. Summary of the theories used in the included studies.

\begin{tabular}{|c|c|c|}
\hline Theory & Medication Therapy & Study \\
\hline ABC Taxonomy and Three Factor Model & Hypertension, heart disease, COPD, asthma & Maffoni et al., 2020 \\
\hline Andersen's Behavioural Model & $\begin{array}{l}\text { Antiretroviral therapy } \\
\text { Antiretroviral therapy }\end{array}$ & $\begin{array}{l}\text { Holtzman et al., } 2015 \\
\text { Schatz et al., } 2019\end{array}$ \\
\hline $\begin{array}{l}\text { Common-Sense Model of } \\
\text { Self-Regulation (CSM) }\end{array}$ & Glaucoma medication & McDonald et al., 2019 \\
\hline Dowell's Therapeutic Alliance & Cardio-protective medication & Lambert-Kerzner et al., 2015 \\
\hline $\begin{array}{l}\text { Dowell's Therapeutic Alliance Model } \\
\text { and Leventhal's Common-Sense Model }\end{array}$ & Use of prescription medicines in general & Kucukarslan et al., 2012 \\
\hline Health Belief Model & $\begin{array}{l}\text { Heart medication } \\
\text { Clopidogrel } \\
\text { Rheumatoid arthritis } \\
\text { Hypertension }\end{array}$ & $\begin{array}{c}\text { Garavalia et al., } 2009 \\
\text { Garavalia et.al., } 2011 \\
\text { Oshotse et.al., } 2018 \\
\text { Rahmawati et al., } 2018\end{array}$ \\
\hline Health Literacy Pathway Model & Diabetes type 2 medication & Huang et al., 2020 \\
\hline $\begin{array}{l}\text { Information-Motivation-Behaviour } \\
\text { Skills (IMB) Model of Adherence }\end{array}$ & Chronic hepatitis $C$ therapy & Evon et al., 2015 \\
\hline Naturalistic Decision-making Model & Heart failure & Meraz et.al., 2020 \\
\hline Roy Adaptation Model & Diabetes type 2 medication & Bockwold et al., 2017 \\
\hline Social Ecological Model & $\begin{array}{l}\text { Cardiovascular medication } \\
\text { Antiretrovial therapy }\end{array}$ & $\begin{array}{l}\text { Petterssen et al., } 2018 \\
\text { Becker et al., } 2020\end{array}$ \\
\hline Stages of Change Model & Anti-diabetic medication & Sapkota et al., 2018 \\
\hline
\end{tabular}

\subsection{Barriers to Medication Adherence}

Overall, the studies reported more barriers than facilitators to medication adherence. We inductively identified six subject areas with subcategories related to barriers to medication adherence based on the included qualitative studies $(n=89)$. The classification was data-driven, and we compiled it after extracting evidence from a third of the studies. We then went back to the beginning and re-extracted the data with the improved categorization. See Figures 2 and 3.

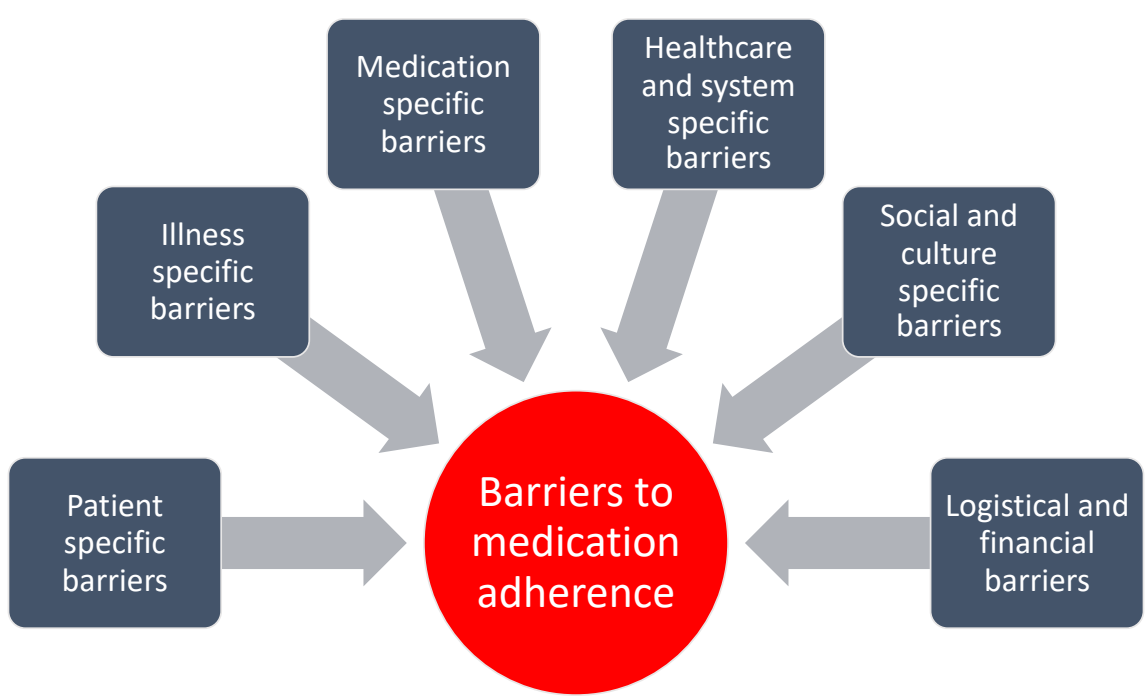

Figure 2. The identified key barriers to medication adherence based on the included qualitative studies $(n=89)$. 


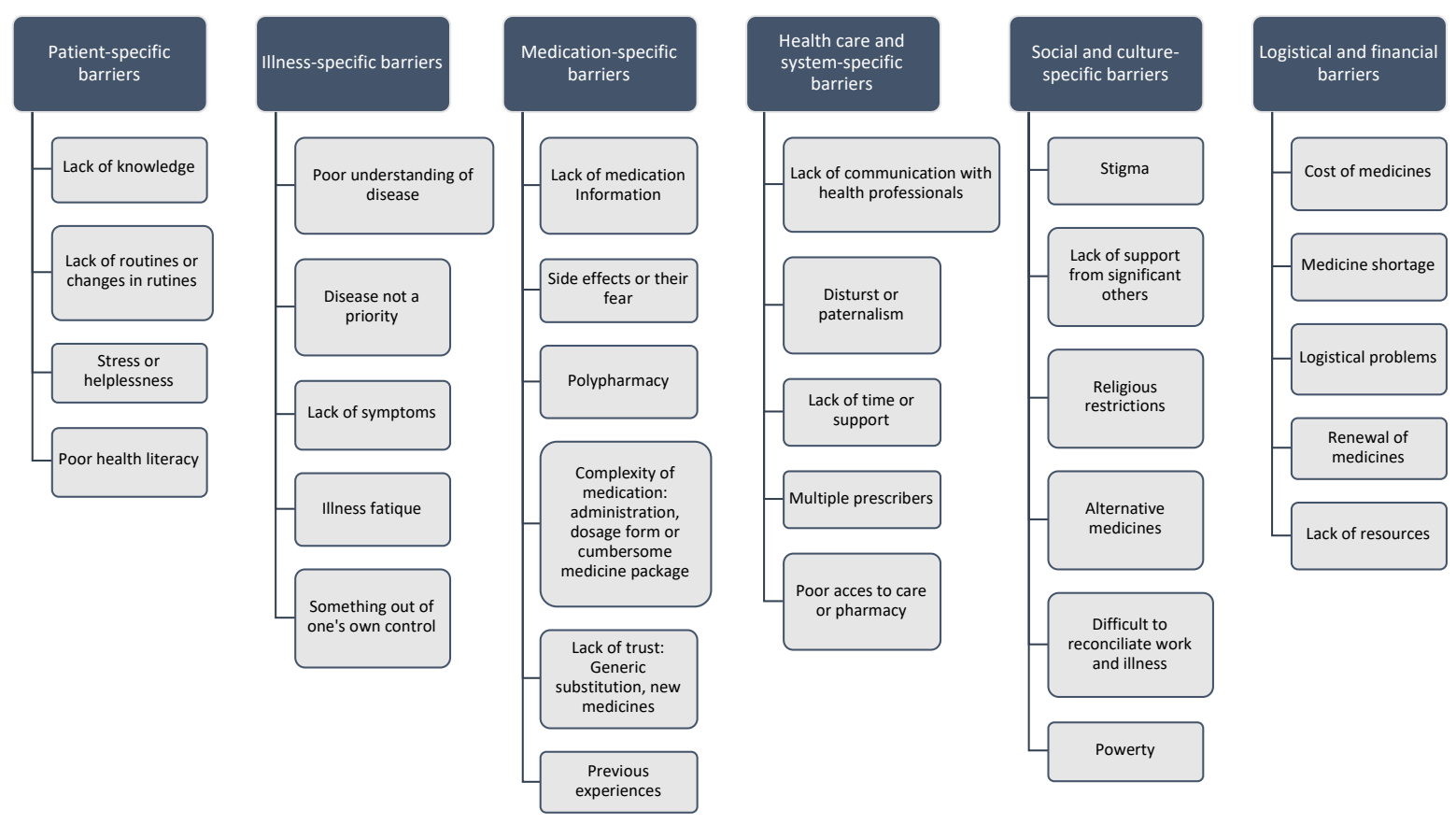

Figure 3. Subcategorisation of barriers to medication adherence arising from the included qualitative studies $(n=89)$.

\subsubsection{Patient-Specific Barriers}

Patients may lack information or knowledge to understand their medication regimen properly. At the beginning of their disease, they may have received medication information and adherence counselling but without any follow-up, leading to patients being forgetful [49]. If the patient is extremely ill at the time of counselling, it may be challenging to adapt the information provided, and misunderstandings can occur. Patients can have poor awareness about the need to take medication as prescribed, and they tend to adjust their doses according to their understanding $[46,50]$. They may have incorrect or erroneous beliefs about medication [51]. They can lack motivation and think the disease is something they cannot control [52]. A lack of routines, being busy, or changes in practices for special occasions are risk points for medication adherence and can easily lead to missing doses or sleeping through dosing times [48].

Stress and helplessness can affect medication adherence [53]. Injectable drugs may feel unpleasant, and a patient may think injecting will destroy the body [52]. Patients' physical disabilities can also be a barrier when administrating the medicine, which may require good eyesight or a steady hand [54]. Poor health literacy increases the adherence problem, and there can also be difficulties in understanding written language, especially if it is not written in a patient's mother tongue [34,41]. Comorbidity may increase the probability of non-adherence [55].

\subsubsection{Illness-Specific Barriers}

Contrary to healthcare professionals' expectations, the disease is not always the priority for the patient $[35,52,56]$. It can be an unwanted episode, but not as important as other matters in life. A patient may have an adverse emotional reaction to the illness and judge life before the illness as more valuable. The required life changes may not be a priority. Patients may also rationalise that the disease is not so severe that they need to take their medication precisely as prescribed. Choosing to take or not to take medicines may depend on how seriously the patient assesses their situation [57].

Sometimes the challenge is that the patient has not accepted the illness or thinks it is someone else's fault. The negative beliefs of illness or multiple diseases can increase barriers to medication adherence though it can differ from condition to condition [58]. Cancer can be understood as more life-threatening than diabetes, although diabetes can 
have grave consequences when not treated as required. The disease itself can cause fatigue and overwhelming tiredness, which negatively impact adherence [59].

\subsubsection{Medication-Specific Barriers}

At the time of the onset of the illness, patients may lack the information on their condition or on the medication they need $[60,61]$. They can feel confused about the illness duration and prognosis $[42,43]$. Treatment can often seem time-consuming and complex to them $[58,62]$. Taking medication can be associated in patients' minds with being sick, which can negatively influence adherence [35]. Difficulties in integrating medication into daily life can prevent patients from taking medication as prescribed. Working life may require shift work, and night shifts may make it difficult to have regular routines [63]. Besides, the illness may not have visible symptoms, and patients may not feel unwell [64]. Patients also fear that once they start a medication, this means they must continue taking it throughout their life [65].

If the medication information for a patient is inadequate and does not meet patients' needs, they may use alternative information sources such as the internet [66]. A patient information leaflet in a medicine package may be difficult to understand. Warnings of side effects in the package sometimes make a patient decide not to take the medicine. Generic substitution may cause suspicions of the effect of a generic drug compared with the original product, thereby negatively affecting adherence [37]. Media can also influence opinions of the quality of drugs [67]. The desire of patients to self-regulate their lives may sometimes lead them to use non-prescription drugs instead of prescribed medicines [68].

Struggling with side effects seems to be a common barrier to medication adherence. Fear and the thought of not being safe with their medication may keep patients from taking it [60]. There are also physical barriers surrounding medication-taking: the size of the tablet can make it difficult to swallow, there can be unpleasant metallic after-taste or throat pain [59]. Needle phobia can prevent injecting insulin. A change from oral tablets to injectable drugs can be a drawback for patients [35].

\subsubsection{Healthcare and System-Specific Barriers}

Poor access to healthcare and long waiting times cause poor medication adherence [55]. Fragmentation of treatment between multiple prescribers, a lack of communication between a general practitioner and a community pharmacist and poor coordination between primary and secondary care can lead to treatment problems. These, in turn, can lead to the discontinuation of care $[55,69,70]$.

A lack of support and empathy from healthcare providers and a paternalistic manner can negatively impact adherence [14,43,55,71]. Poor patient-provider relationships lead to insufficient patient counselling and leave the patient alone struggling with medication problems [43]. Without trust-based patient-provider communication, patients cannot freely discuss side effects and other concerns related to their medication $[39,72]$. The inability of healthcare professionals to discuss adherence problems with patients and take their concerns and experiences seriously can impact the self-efficacy of patients [73,74]. A lack of trust in doctors and questioning their expertise may increase the burden of the illness and have an essential influence on a patient's adherence behaviour [60].

\subsubsection{Social and Culture-Specific Barriers}

A stigma is a common reason for nonadherence, especially with HIV / AIDS and with non-communicable diseases [71]. Patients may not want anybody to know about their illness. The fear of being stigmatized can be so intense that the patient prefers not to take their medication if there is a possibility that someone might be watching. It can be difficult to reconcile work and illness [74]. A lack of support from significant others can have a substantial impact on adherence and control of the illness [75,76].

Patients can prefer traditional alternatives or homeopathic remedies or methods instead of conventional medicine because these are more "natural" [45,72,77]. Patients can 
have a strong religious faith and prioritize religious rituals instead of taking medicines. Fasting during Ramadan and holy water can have a significant impact on medication management and may be the leading cause to adjust the medication to fit better with religious situations and routines [71]. Patients may stop the medication if they believe that praying can cure them [78].

\subsubsection{Logistical and Financial Barriers}

Financial burdens and costs of medicines are significant barriers to medication adherence [79]. Unemployment and economic difficulties can affect the ability to buy medicines. If a patient does not have enough money to buy necessities such as food and clothing, medicines are unlikely to be a priority [80]. Difficulties travelling to the clinic, especially in developing countries, can hinder good medication self-management [42]. If insurance coverage is not comprehensive enough or there is no insurance, the cost of medicine can be unbearable [80]. A medicine shortage and the availability of medicines at the clinic or pharmacy, especially in developing countries, can become a significant problem for the continuity of care [60].

\subsection{Facilitators to Medication Adherence}

We identified five subject areas related to facilitators of medication adherence. Because medication taking is related to individual behaviour, we used the Information-MotivationBehavioural Skills (IMB) Model as a starting point for the analysis $[15,16]$. However, as medication adherence is a complex entity in addition to human behaviour, we observed healthcare and system-specific factors and logistical and financial factors (Figures 4 and 5).

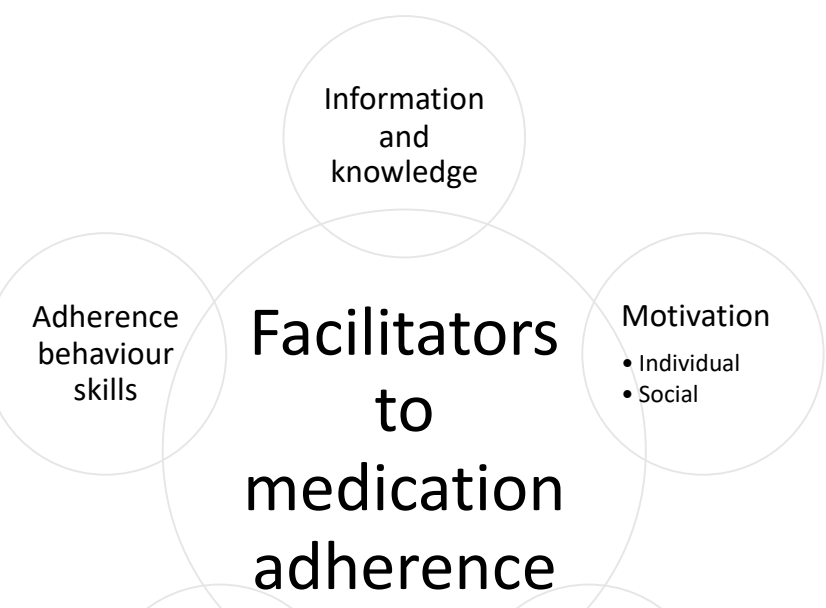

Healthcare
and system
spesific
factors

$$
\begin{aligned}
& \text { Logistical } \\
& \text { and } \\
& \text { financial } \\
& \text { factors }
\end{aligned}
$$

Figure 4. The identified key facilitators to medication adherence based on the included qualitative studies $(n=89)$. 


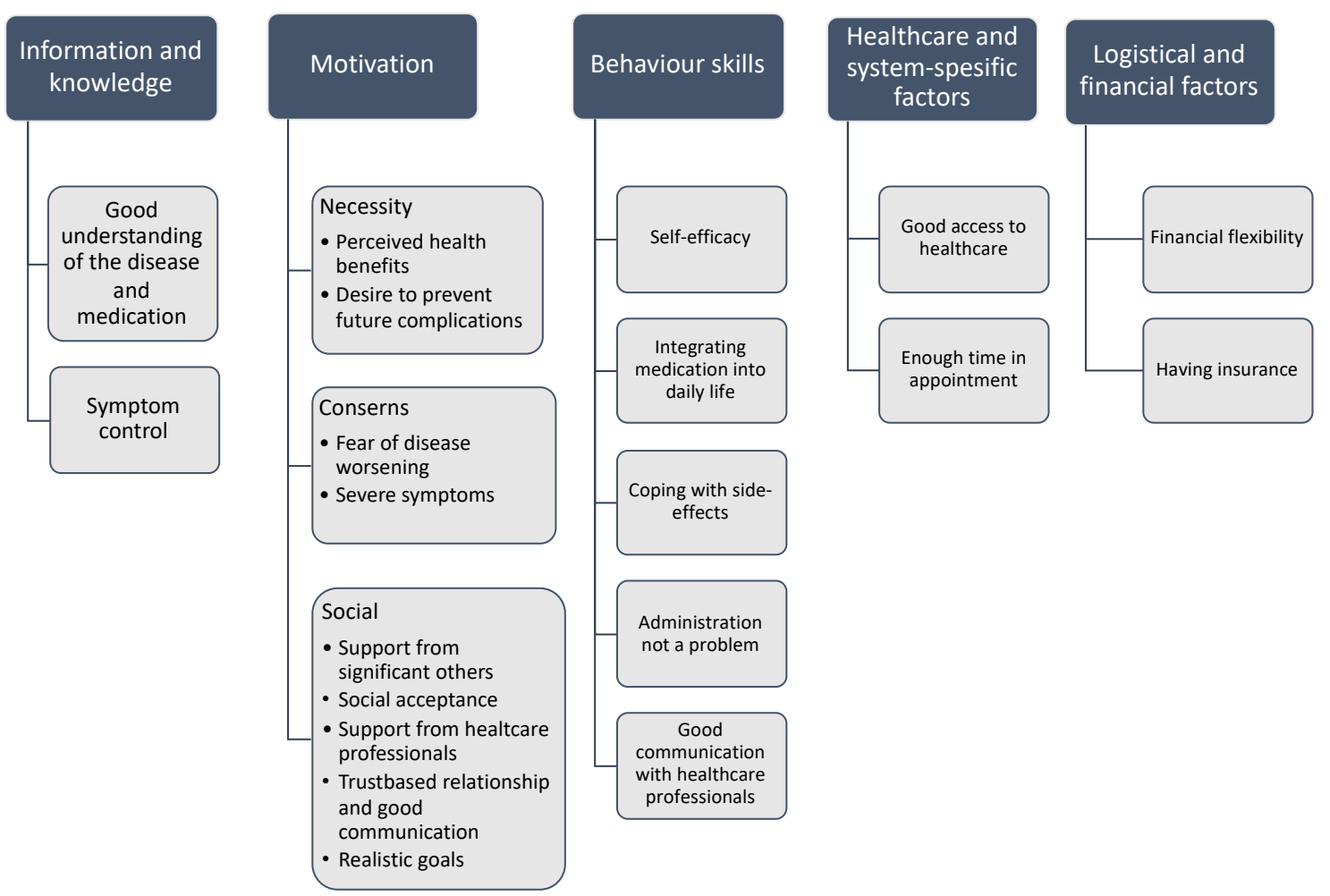

Figure 5. Categories and subcategories of facilitators to medication adherence arising from the included qualitative studies $(n=89)$. We used the Information-Motivation-Behaviour skills (IMB) model as part of the classification.

\subsubsection{Informational, Motivational and Behavioural Factors}

A good understanding of the illness and its treatment and how medicines promote the quality of life is essential for adherence [68]. The ability to integrate medications into daily life improves adherence in self-managing chronic conditions [79]. Low toxicity, mild adverse effects and an oral route of the administration seem to promote medication adherence $[66,79]$. There are different tools to assist with medicine taking, such as pillboxes, clock or mobile alarms, or taking medications during regular TV and radio programs [49,55].

The patient's motivation is an essential facilitator. Motivation improves if the patient understands the necessity of the medication, and it contributes to positive health benefits [80]. Significant life events can have a positive effect on medication adherence. If a serious complication occurs, the importance of preventing complications and maintaining health is highlighted and may lead to a re-evaluation of the patient's priorities [35,64,81]. The desire to return to "normal life" is a powerful facilitator to medication adherence [53].

The concerns related to illness may improve adherence and motivation to take medication as prescribed $[79,82]$. If patients have lived through the experience of their disease and its further negative impact on functional abilities, medication adherence may increase [82]. Knowing that interrupting or changing medications would result in the disease worsening can increase the desire to self-manage medication better [79]. The treatment goals must be realistic and achievable for the patient [52].

Support from family and friends and colleagues at work support adherence. It may require the disclosure of the illness, which can be scary for the patient [60,71]. Social acceptance helps the patient to cope with the illness.

Self-efficacy is an essential skill when coping with practical problems in daily life. If the patient takes ownership of self-managing the medication and knows how to adjust medicines if the disease worsens, the chances for better adherence are higher $[60,83]$. Feeling responsible and having a strong belief in the efficacy of medication promote selfempowerment and create a positive attitude towards the medication [59]. 


\subsubsection{Healthcare and System-Specific Facilitators}

A trust-based, collaborative and respectful patient-provider relationship is crucial for medication adherence [55]. Good access to healthcare and enough time for discussions are necessary for patients [57]. Sometimes a desire to please healthcare providers or fearing them may also facilitate adherence [55]. Patients wish for confidential communication and an ongoing dialogue with health care professionals [84]. Support from healthcare providers and freely accessible care appear to increase adherence [65].

\subsubsection{Logistical and Financial Factors}

Financial flexibility is necessary for medication adherence. The balance between revenue and expenditure of the household makes it possible to buy essential commodities such as food, clothes, and medicines without prioritising [80]. Additionally, having good insurance coverage guarantees secure finances in contrast to having no insurance at all.

\subsection{Summary of Findings}

We identified an extensive range of barriers and facilitators to medication adherence and the studies were more often concerned with barriers than facilitators. We classified the barriers as patient-specific, illness-specific, medication-related, healthcare and systemrelated, sociocultural and logistical and financial factors. The facilitators we identified were information and knowledge of the disease and medication, individual and social motivation, adherence behaviour skills, healthcare and system-specific factors and logistical and financial factors. Some of these factors can act as barriers and facilitators, such as healthcare and system-related factors and logistical and financial factors.

We identified similar factors to medication adherence in the previous systematic qualitative reviews ( $n=13$, Table A2) as in the qualitative studies described above $(n=76$, Table A1). The previous systematic review findings confirm our own findings and the complexity of medication adherence as a phenomenon.

Some of the included studies had a theory-based approach to medication adherence $(n=17)$ (Table 1). Using different theories helped to understand and explain patients actions related to taking their medication (Table 1).

\section{Discussion}

\subsection{Main Findings}

To improve medication adherence, better communication and better information on the disease and its medication appeared to be the crucial concepts for patients in this scoping review. Our findings confirm that medication adherence is a complex phenomenon that is only partly understood. A wide range of factors seems to influence this either positively or negatively or in both ways. Regardless of the study concept, our findings were similar from study to study. Patients have many concerns about their illness, and it seems that they do not commonly have enough information to make knowledge-based decisions for self-managing their care. Patients want to discuss their problems and fears with a healthcare provider, but there is often not enough time for that in a short appointment.

According to this scoping review, the illness was not always a priority for the person. There can be many other matters in life that people prioritise more than their own optimal disease self-management. For better medication adherence, healthcare providers need to pay more attention to patients' thoughts and concerns and have more time to listen to their experience in relation to the disease. Patients highly value trust-based relationships with healthcare providers.

This scoping review tracked many barriers that can hinder patients' intention to adhere to their medication taking. The complexity of the matter may explain why many interventions to improve medication adherence are not successful [3]. If the intervention targets only some of the barriers, positive outcomes may be lacking, despite good intentions. The adherence to one medicine does not either automatically mean adherence to other medicines. Thus, adherence can differ from treatment to treatment or from disease to 
disease [68]. Patients may make their own priorities about the medications they use. This phenomenon should be further researched.

Our review of qualitative studies indicates that more attention should be paid to the patients' fear of side effects. This can be a barrier that affects medication taking and can lead to skipping doses. With good knowledge and open and trust-based discussion with a healthcare provider, the patient need not begin to doubt their treatment. It is also good to discuss the patient's values and religious values. A well-informed patient should know how to adjust medication to fit with religious requirements. The better the healthcare providers know the reality of their patients; living situations, the better they can support their patients to become empowered to self-manage a complex medication regimen.

Barriers can exist that the healthcare provider has not taken into consideration. The patient may have obstacles to self-manage taking their medication, for example, the difficulties of injecting medicines, remembering to take their medication on time when working, or the fear of stigmatisation. Additionally, financial obstacles can be difficult to reveal. Ideally, health care professionals should meet the patient without any preconceptions and in a trusted environment to discuss the barriers and concerns related to medication.

A theory-based approach may help to understand the patient's actions and behaviours. However, a minority of the research we found had a theoretical approach, and the theories applied varied. Different behavioural theories, also adherence-specific ones, aim to explain chronically ill patient's behaviour and give a reasonable explanation of why the patients act as they do. According to those theories, the patient's action depends on their behaviour. This, in turn, depends on the patient's beliefs or expected outcomes. According to our findings, an IMB model explains factors influencing adherence related to patient's behaviour. However, external circumstances affect adherence, such as financial problems or poor access to care, which have to be considered. Based on our analyses, the different behavioural theories are good tools but do not fully explain complex adherence behaviour.

There is a need to generate new theory-based approaches to medication adherence since the current behavioural theories are not completely successful in explaining the complex phenomenon of adherence. There are also numerous adherence scales, which are very diverse and difficult to compare, so the research may need to be focused on comparing existing scales and determining which are most reliable. Qualitative research provides new insights into patient experiences and daily life struggles with their diseases and medicine taking to be incorporated in further development of the adherence measures and models. A good example of such a novel and promising conceptual approach is a model of medication-related burden and patients' lived experiences with medicine, which builds on a meta-synthesis of qualitative studies [12].

Our review covered 13 systematic reviews, of which 11 were disease-specific, and 2 were generic. The illness-specific systematic reviews pointed out that patients often had misinterpretations of their illness, which prevented their adherence to medication. Clarifying these issues, having time and support, including from family members, were key recommendations to improve adherence in these reviews.

In our findings, there were more studies on barriers to medication adherence than facilitators. This is an interesting finding and can be affected by the fact that medication adherence is poorly understood, at least how it can be improved. When trying to understand the patient's struggle with complex medication regimens, it may have caused the focus of research to go more towards barriers than facilitators. This scoping review may help to better understand the broader picture of adherence and to find interventions and strategies to improve it.

To our knowledge, this is the first scoping review on patient-related factors of medication adherence based on qualitative research. We conclude that well-informed patients and trustful patient-provider relationships are at the centre of improving medication adherence. Self-efficacy is crucial and empowers the patient to control and self-manage the disease and adjust the medication when necessary. Patient motivation needs to be monitored and supported. Moreover, patients need help integrating the medication regimen into their 
daily lives and to have routines. Support from significant others is essential too. They can support the patient in a life-long journey with the disease and give motivation for good medication self-management.

However, more research is needed to understand the patient's reality. This scoping review clarifies the contributing factors of nonadherence and why the outcomes of interventions to improve adherence can be poor. The observations presented in this scoping review are useful when planning more effective interventions to increase medication adherence.

\subsection{Strengths and Limitations}

This scoping review of qualitative studies provides new information on people's medicine-taking behaviour, which may not have been used to the best advantage. The strengths of this scoping review include an extensive literature search and review, followed by a thorough categorization of the barriers and facilitators of medication adherence. The literature searches were made with the support of an experienced librarian, and we had good coverage of qualitative studies where the primary focus was patients' experiences and attitudes towards medication adherence. To avoid a selection bias, there were three researchers involved in the selection process. The data was thoroughly extracted and analysed to define the overarching categories.

A limitation is that since we focused on the qualitative aspects, we cannot conclude the magnitude of the effect of several factors influencing adherence. We also limited our search to studies in English, which may be a source of bias. The studies reported more barriers than facilitators, which may be another limitation. On the other hand, it describes the fact that barriers have been better recognized than facilitators. More research should be focused on the factors that have been able to help patients to commit to their disease and medication self-management. More research is also needed to elaborate on new theoretical models. This scoping review provides a good basis for building up more comprehensive theoretical models on medication adherence.

\section{Conclusions}

This scoping review highlighted a wide range of barriers and facilitators. The barriers seem to be better known than the facilitators. There is a need for better recognition of facilitators. We may need to increase the qualitative research of medication adherence to better understand the patients lived experiences that direct their medicine-taking behaviour. This information is needed to find new interventions and approaches to increase medication adherence, compare existing adherence scales, and build up more comprehensive theoretical models on medication adherence.

Patients wish to discuss their concerns about medications. Better communication and information appear to be among the most crucial factors for patients. The factors presented in this scoping review may help clinicians who communicate with patients having issues with adherence. The findings of this scoping review may also help those who plan further interventions to build up a more comprehensive approach to improve medication adherence.

Author Contributions: Conceptualization, K.K., M.A. and H.L.; methodology, K.K., A.W., M.A. and H.L.; formal analysis, K.K. and H.L.; investigation, K.K. and H.L.; writing-original draft preparation, K.K.; writing-review and editing, K.K., A.W., M.A. and H.L.; visualization, K.K.; supervision, M.A. and H.L.; project administration, K.K.; funding acquisition, K.K. All authors have read and agreed to the published version of the manuscript.

Funding: This research was funded by the Foundation for Municipal Development (personal grant for Kirsi Kvarnström). Open access funding provided by University of Helsinki.

Institutional Review Board Statement: Not applicable (literature review).

Informed Consent Statement: Not applicable. 
Data Availability Statement: The available data is included in Appendices A-C. Other data used in this study are available on request from the corresponding author.

Acknowledgments: We would like to thank the Information Specialist Terhi Sandgren at the Helsinki University Library for assistance in the systematic literature search.

Conflicts of Interest: The authors declare no conflict of interest. The funders had no role in the design of the study; in the collection, analyses, or interpretation of data; in the writing of the manuscript, or in the decision to publish the results.

\section{Appendix A}

Search Strategy for the Scopus Database

(TITLE-ABS-KEY ((medication * OR drug * OR medicine $\left.{ }^{*}\right)$ W/2 (adhere * OR nonadheren * OR nonadheren * OR complian * OR noncomplian *))) AND (TITLE-ABS-KEY (patient ${ }^{*} \mathrm{~W} / 2$ (experienc ${ }^{*} \mathrm{OR}$ fear ${ }^{*} \mathrm{OR}$ belie * OR knowled * OR attitude OR behavio * OR communicat * OR reason OR reasons OR cause ${ }^{*}$ ))) AND (TITLE-ABS-KEY (qualitative OR interview * OR "focus group *" OR questionnaire * OR (observation * W/2 (study OR research)))) AND (LIMIT-TO (PUBYEAR, 2019) OR LIMIT-TO (PUBYEAR, 2018) OR LIMIT-TO (PUBYEAR, 2017) OR LIMIT-TO (PUBYEAR, 2016) OR LIMIT-TO (PUBYEAR, 2015) OR LIMIT-TO (PUBYEAR, 2014) OR LIMIT-TO (PUBYEAR, 2013) OR LIMIT-TO (PUBYEAR, 2012) OR LIMIT-TO (PUBYEAR, 2011) OR LIMIT-TO (PUBYEAR, 2010) OR LIMIT-TO (PUBYEAR, 2009))

\section{Appendix B}

Table A1. Summary of included studies.

\begin{tabular}{|c|c|c|c|c|c|c|}
\hline Study & Title & Study Design & Concept & Context & Illness & $\begin{array}{l}\text { Country in } \\
\text { Which the } \\
\text { Study } \\
\text { Conducted }\end{array}$ \\
\hline $\begin{array}{l}\text { Al-Qazaz et al., } \\
2011\end{array}$ & $\begin{array}{l}\text { Perception and } \\
\text { knowledge of } \\
\text { patients with } \\
\text { type 2diabetes } \\
\text { in Malaysia } \\
\text { about their } \\
\text { disease and } \\
\text { medication: A } \\
\text { qualitative } \\
\text { study }\end{array}$ & $\begin{array}{l}\text { Individual } \\
\text { interviews }\end{array}$ & $\begin{array}{c}\text { Diabetic' } \\
\text { patients' } \\
\text { experience and } \\
\text { knowledge } \\
\text { about diabetes } \\
\text { and its } \\
\text { medication and } \\
\text { the factors } \\
\text { contributing to } \\
\text { medication } \\
\text { adherence }\end{array}$ & $\begin{array}{c}\text { Diabetic } 2 \\
\text { patients' } \\
\text { adherence to } \\
\text { medication and } \\
\text { knowledge } \\
\text { about their } \\
\text { illness }\end{array}$ & $\begin{array}{c}\text { Cardiovascular } \\
\text { disease }\end{array}$ & Malaysia \\
\hline $\begin{array}{l}\text { AlHamid et al., } \\
2014\end{array}$ & $\begin{array}{l}\text { A systematic } \\
\text { review of } \\
\text { qualitative } \\
\text { research on the } \\
\text { contributory } \\
\text { factors leading } \\
\text { to medicine- } \\
\text { related } \\
\text { problems from } \\
\text { the perspectives } \\
\text { of adult } \\
\text { patients with } \\
\text { cardiovascular } \\
\text { diseases and } \\
\text { diabetes } \\
\text { mellitus }\end{array}$ & $\begin{array}{l}\text { Systematic } \\
\text { review }\end{array}$ & $\begin{array}{c}\text { To explore and } \\
\text { evaluate } \\
\text { contributory } \\
\text { factors leading } \\
\text { to MRPs among } \\
\text { adult patients } \\
\text { with CVDs } \\
\text { and/or DM } \\
\text { from their } \\
\text { perspectives }\end{array}$ & $\begin{array}{l}\text { Outpatients } \\
\text { with diabetes or } \\
\text { cardiovascular } \\
\text { diseases }\end{array}$ & $\begin{array}{c}\mathrm{DM} \text { and } \\
\text { cardiovascular } \\
\text { disease }\end{array}$ & $\begin{array}{l}12 \text { countries: } \\
\text { Australia, } \\
\text { Brazil, } \\
\text { Cameroon, } \\
\text { Canada, } \\
\text { Croatia, Ireland, } \\
\text { Malaysia, South } \\
\text { Africa, Spain, } \\
\text { Taiwan, the UK, } \\
\text { and the USA }\end{array}$ \\
\hline
\end{tabular}


Table A1. Cont.

\begin{tabular}{|c|c|c|c|c|c|c|}
\hline Study & Title & Study Design & Concept & Context & Illness & $\begin{array}{l}\text { Country in } \\
\text { Which the } \\
\text { Study } \\
\text { Conducted }\end{array}$ \\
\hline Ali et al., 2019 & $\begin{array}{l}\text { Qualitative } \\
\text { Analysis of } \\
\text { Factors } \\
\text { Influencing } \\
\text { Patient } \\
\text { Persistence and } \\
\text { Adherence to } \\
\text { Prescribed } \\
\text { Overactive } \\
\text { Bladder } \\
\text { Medication in } \\
\text { UK Primary } \\
\text { Care }\end{array}$ & $\begin{array}{l}\text { Individual } \\
\text { interviews }\end{array}$ & $\begin{array}{l}\text { Non- } \\
\text { Adherence has } \\
\text { a major impact } \\
\text { on health } \\
\text { outcomes in } \\
\text { long term } \\
\text { diseases. }\end{array}$ & $\begin{array}{c}\text { Primary care in } \\
\text { the UK }\end{array}$ & $\begin{array}{l}\text { Overactive } \\
\text { bladder }\end{array}$ & UK \\
\hline $\begin{array}{l}\text { Alodhaib et al., } \\
\qquad 2021\end{array}$ & $\begin{array}{c}\text { Qualitative } \\
\text { Exploration of } \\
\text { Barriers to } \\
\text { Medication } \\
\text { Adherence } \\
\text { Among Patients } \\
\text { with } \\
\text { Uncontrolled } \\
\text { Diabetes in } \\
\text { Saudi Arabia }\end{array}$ & $\begin{array}{l}\text { Individual } \\
\text { interviews }\end{array}$ & $\begin{array}{c}\text { Physicians can } \\
\text { rarely identify } \\
\text { non-adherent } \\
\text { patients }\end{array}$ & $\begin{array}{c}\text { Patients at a } \\
\text { diabetes centre } \\
\text { in Saudi-Arabia }\end{array}$ & DM & Saudi-Arabia \\
\hline $\begin{array}{l}\text { Alhomoud } \\
\text { et al., } 2015\end{array}$ & $\begin{array}{l}\text { South Asian, } \\
\text { and Middle } \\
\text { Eastern } \\
\text { patients' } \\
\text { perspectives on } \\
\text { medicine- } \\
\text { related } \\
\text { problems in the } \\
\text { United } \\
\text { Kingdom }\end{array}$ & $\begin{array}{l}\text { Individual } \\
\text { interviews }\end{array}$ & $\begin{array}{l}\text { Medication } \\
\text { adherence }\end{array}$ & $\begin{array}{l}\text { Outpatients in } \\
\text { community } \\
\text { pharmacies in } \\
\text { London }(n=94)\end{array}$ & $\begin{array}{l}\text { Not mentioned } \\
\text { (patients in } \\
\text { general) }\end{array}$ & UK \\
\hline $\begin{array}{l}\text { Axelsson et al., } \\
2015\end{array}$ & $\begin{array}{l}\text { Antiretroviral } \\
\text { therapy } \\
\text { adherence } \\
\text { strategies used } \\
\text { by patients of a } \\
\text { large HIV clinic } \\
\text { in Lesotho }\end{array}$ & $\begin{array}{l}\text { Individual } \\
\text { interviews }\end{array}$ & $\begin{array}{c}\text { Adherence to } \\
\text { ART } \\
\text { (outpatients) }\end{array}$ & $\begin{array}{c}\text { Patients } \\
\text { receiving ART } \\
\text { in their monthly } \\
\text { clinical visit }\end{array}$ & HIV / AIDS & Lesotho \\
\hline $\begin{array}{l}\text { Barasa Masaba } \\
\text { et al., } 2020\end{array}$ & $\begin{array}{l}\text { Determinants } \\
\text { of Non- } \\
\text { Adherence to } \\
\text { Treatment } \\
\text { Among Patients } \\
\text { with Type } 2 \\
\text { Diabetes in } \\
\text { Kenya: A } \\
\text { Systematic } \\
\text { Review. } \\
\text { (00Review) }\end{array}$ & $\begin{array}{l}\text { Systematic } \\
\text { review }\end{array}$ & $\begin{array}{l}\text { Diabetes is the } \\
\text { leading non- } \\
\text { communicable } \\
\text { disease in } \\
\text { Kenya }\end{array}$ & $\begin{array}{l}\text { Health care in } \\
\text { Kenya }\end{array}$ & DM2 & Kenya \\
\hline
\end{tabular}


Table A1. Cont.

\begin{tabular}{|c|c|c|c|c|c|c|}
\hline Study & Title & Study Design & Concept & Context & Illness & $\begin{array}{l}\text { Country in } \\
\text { Which the } \\
\text { Study } \\
\text { Conducted }\end{array}$ \\
\hline $\begin{array}{l}\text { Becker et al., } \\
2020\end{array}$ & $\begin{array}{c}\text { Individual, } \\
\text { household, and } \\
\text { community } \\
\text { level barriers to } \\
\text { ART adherence } \\
\text { among women } \\
\text { in rural } \\
\text { Eswatini }\end{array}$ & Focus group & $\begin{array}{l}\text { Barriers to ART } \\
\text { among woman } \\
\text { living with HIV } \\
\text { in communities }\end{array}$ & $\begin{array}{l}\text { Rural women } \\
\text { living with } \\
\text { HIV / AIDS }\end{array}$ & HIV / AIDS & Eswatini, Africa \\
\hline $\begin{array}{l}\text { Bezabhe et al., } \\
2014\end{array}$ & $\begin{array}{c}\text { Barriers and } \\
\text { Facilitators of } \\
\text { Adherence to } \\
\text { Antiretroviral } \\
\text { Drug Therapy } \\
\text { and Retention } \\
\text { in Care among } \\
\text { Adult } \\
\text { HIV-Positive } \\
\text { Patients: A } \\
\text { Qualitative } \\
\text { Study from } \\
\text { Ethiopia }\end{array}$ & $\begin{array}{l}\text { Individual } \\
\text { interviews }\end{array}$ & $\begin{array}{c}\text { Adherence to } \\
\text { ART }\end{array}$ & $\begin{array}{l}\text { Outpatients } \\
\text { receiving ART } \\
\text { in HIV-clinic, } \\
\text { problems in } \\
\text { medication } \\
\text { taking }\end{array}$ & HIV / AIDS & Ethiopia \\
\hline $\begin{array}{l}\text { Bockwold et al., } \\
2017\end{array}$ & $\begin{array}{l}\text { Understanding } \\
\text { experiences of } \\
\text { diabetes } \\
\text { medications } \\
\text { among African } \\
\text { Americans } \\
\text { living with type } \\
2 \text { diabetes }\end{array}$ & $\begin{array}{l}\text { Individual } \\
\text { interviews }\end{array}$ & $\begin{array}{l}\text { The } \\
\text { contributing } \\
\text { factors to } \\
\text { non-adherence } \\
\text { to diabetic } \\
\text { medications in } \\
\text { AAs }\end{array}$ & $\begin{array}{l}\text { Hospital-based } \\
\text { outpatient } \\
\text { diabetes clinic } \\
\text { in low-income } \\
\text { Chicago }\end{array}$ & DM2 & United States \\
\hline $\begin{array}{l}\text { Chen et al., } \\
2014\end{array}$ & $\begin{array}{c}\text { Disease } \\
\text { acceptance and } \\
\text { adherence to } \\
\text { imatinib in } \\
\text { Taiwanese } \\
\text { chronic } \\
\text { myeloid } \\
\text { leukaemia } \\
\text { outpatients }\end{array}$ & $\begin{array}{l}\text { Individual } \\
\text { interviews }\end{array}$ & $\begin{array}{l}\text { Adherence to } \\
\text { imatinib, a } \\
\text { medication for } \\
\text { CML }\end{array}$ & $\begin{array}{c}\text { Outpatient } \\
\text { clinic in Taiwan }\end{array}$ & $\begin{array}{l}\text { Chronic } \\
\text { myeloid } \\
\text { leukaemia }\end{array}$ & Taiwan \\
\hline $\begin{array}{c}\text { Clancy et al., } \\
2020\end{array}$ & $\begin{array}{l}\text { Breast cancer } \\
\text { patients' } \\
\text { experiences of } \\
\text { adherence and } \\
\text { persistence to } \\
\text { oral endocrine } \\
\text { therapy: A } \\
\text { qualitative } \\
\text { evidence } \\
\text { synthesis }\end{array}$ & $\begin{array}{l}\text { Systematic } \\
\text { review }\end{array}$ & $\begin{array}{l}\text { Breast cancer } \\
\text { patients' } \\
\text { experiences of } \\
\text { adherence }\end{array}$ & $\begin{array}{l}\text { Outpatient } \\
\text { setting }\end{array}$ & Breast cancer & Ireland \\
\hline
\end{tabular}


Table A1. Cont.

\begin{tabular}{|c|c|c|c|c|c|c|}
\hline Study & Title & Study Design & Concept & Context & Illness & $\begin{array}{l}\text { Country in } \\
\text { Which the } \\
\text { Study } \\
\text { Conducted }\end{array}$ \\
\hline $\begin{array}{l}\text { Dehdari et al., } \\
2019\end{array}$ & $\begin{array}{c}\text { The } \\
\text { determinants of } \\
\text { anti-diabetic } \\
\text { medication } \\
\text { adherence } \\
\text { based on the } \\
\text { experiences of } \\
\text { type } 2 \text { diabetes }\end{array}$ & $\begin{array}{l}\text { Individual } \\
\text { interviews }\end{array}$ & $\begin{array}{l}\text { Medication } \\
\text { adherence as } \\
\text { presented by } \\
\text { type } 2 \mathrm{DM} \\
\text { patients and } \\
\text { their families }\end{array}$ & $\begin{array}{l}\text { Secondary care } \\
\text { outpatient } \\
\text { clinic in Iran }\end{array}$ & DM2 & Iran \\
\hline $\begin{array}{c}\text { Eliasson et al., } \\
2011\end{array}$ & $\begin{array}{c}\text { Exploring } \\
\text { chronic } \\
\text { myeloid } \\
\text { leukaemia } \\
\text { patients' } \\
\text { reasons for not } \\
\text { adhering to the } \\
\text { oral anticancer } \\
\text { drug imatinib } \\
\text { as prescribed }\end{array}$ & $\begin{array}{l}\text { Individual } \\
\text { interviews }\end{array}$ & $\begin{array}{c}\text { CML } \\
\text { outpatients } \\
\text { who have been } \\
\text { prescribed } \\
\text { imatinib }\end{array}$ & $\begin{array}{c}\text { CML } \\
\text { outpatients } \\
\text { with prescribed } \\
\text { imatinib } \\
\text { medication }\end{array}$ & $\begin{array}{c}\text { Chronic } \\
\text { myeloid } \\
\text { leukaemia }\end{array}$ & United States \\
\hline Evon et al., 2015 & $\begin{array}{c}\text { Adherence } \\
\text { during } \\
\text { Antiviral } \\
\text { Treatment } \\
\text { Regimens for } \\
\text { Chronic } \\
\text { Hepatitis C: A } \\
\text { Qualitative } \\
\text { Study of } \\
\text { Patient- } \\
\text { Reported } \\
\text { Facilitators and } \\
\text { Barriers }\end{array}$ & $\begin{array}{l}\text { Individual } \\
\text { interviews }\end{array}$ & $\begin{array}{l}\text { HCV patients' } \\
\text { adherence } \\
\text { taking HCV } \\
\text { medication as } \\
\text { prescribed }\end{array}$ & $\begin{array}{c}\text { Outpatients } \\
\text { who receive } \\
\text { HCV } \\
\text { medication }\end{array}$ & $\begin{array}{c}\text { Chronic } \\
\text { Hepatitis C }\end{array}$ & United States \\
\hline $\begin{array}{l}\text { Farinha et al., } \\
2017\end{array}$ & $\begin{array}{c}\text { Concerns of } \\
\text { patients with } \\
\text { systemic lupus } \\
\text { erythematosus } \\
\text { and adherence } \\
\text { to therapy-a } \\
\text { qualitative } \\
\text { study }\end{array}$ & $\begin{array}{l}\text { Individual } \\
\text { interviews }\end{array}$ & $\begin{array}{l}\text { SLE outpatients } \\
\text { thoughts and } \\
\text { concerns about } \\
\text { their illness and } \\
\text { medication } \\
\text { management }\end{array}$ & $\begin{array}{l}\text { Outpatient } \\
\text { clinics }\end{array}$ & $\begin{array}{l}\text { Systemic lupus } \\
\text { erythematosus }\end{array}$ & Portugal \\
\hline $\begin{array}{c}\text { Frech et al., } \\
2021\end{array}$ & $\begin{array}{l}\text { Patterns and } \\
\text { facilitators for } \\
\text { the promotion } \\
\text { of glaucoma } \\
\text { medication } \\
\text { adherence-a } \\
\text { qualitative } \\
\text { study }\end{array}$ & $\begin{array}{l}\text { Individual } \\
\text { interviews }\end{array}$ & $\begin{array}{c}\text { Better } \\
\text { understanding } \\
\text { of patient } \\
\text { patterns in } \\
\text { glaucoma } \\
\text { medication } \\
\text { management }\end{array}$ & $\begin{array}{c}\text { Department of } \\
\text { Ophthalmol- } \\
\text { ogy in } \\
\text { Germany }\end{array}$ & Glaucoma & Germany \\
\hline
\end{tabular}


Table A1. Cont.

\begin{tabular}{|c|c|c|c|c|c|c|}
\hline Study & Title & Study Design & Concept & Context & Illness & $\begin{array}{l}\text { Country in } \\
\text { Which the } \\
\text { Study } \\
\text { Conducted }\end{array}$ \\
\hline $\begin{array}{l}\text { Garavalia et al., } \\
2009\end{array}$ & $\begin{array}{c}\text { Exploring } \\
\text { Patients' } \\
\text { Reasons for } \\
\text { Discontinuance } \\
\text { of Heart } \\
\text { Medications }\end{array}$ & $\begin{array}{l}\text { Individual } \\
\text { interviews }\end{array}$ & $\begin{array}{l}\text { To understand } \\
\text { patients' } \\
\text { viewpoints on } \\
\text { why they } \\
\text { stopped taking } \\
\text { their } \\
\text { medication }\end{array}$ & $\begin{array}{l}\text { MI outpatients } \\
\text { who have } \\
\text { stopped to take } \\
\text { their prescribed } \\
\text { medication } \\
\text { (clopidogrel or } \\
\text { cholesterol } \\
\text { lowering } \\
\text { medication) }\end{array}$ & $\begin{array}{c}\text { Cardiovascular } \\
\text { disease }\end{array}$ & United States \\
\hline $\begin{array}{l}\text { Garavalia et al., } \\
2011\end{array}$ & $\begin{array}{c}\text { Clinician- } \\
\text { Patient Discord: } \\
\text { Exploring } \\
\text { Differences in } \\
\text { Perspectives for } \\
\text { Discontinuing } \\
\text { Clopidogrel }\end{array}$ & $\begin{array}{l}\text { Individual } \\
\text { interviews }\end{array}$ & $\begin{array}{c}\text { Why MI } \\
\text { outpatients stop } \\
\text { to take } \\
\text { clopidogrel }\end{array}$ & $\begin{array}{l}\text { Outpatients } \\
\text { setting }\end{array}$ & $\begin{array}{c}\text { Cardiovascular } \\
\text { disease }\end{array}$ & United States \\
\hline $\begin{array}{l}\text { Gassmann et al., } \\
2016\end{array}$ & $\begin{array}{l}\text { Experiences } \\
\text { and coping } \\
\text { strategies of } \\
\text { oncology } \\
\text { patients } \\
\text { undergoing } \\
\text { oral } \\
\text { chemotherapy: } \\
\text { First steps of a } \\
\text { grounded } \\
\text { theory study }\end{array}$ & $\begin{array}{l}\text { Individual } \\
\text { interviews }\end{array}$ & $\begin{array}{l}\text { Patients' } \\
\text { thoughts about } \\
\text { their oral } \\
\text { chemotherapy } \\
\text { management }\end{array}$ & $\begin{array}{l}\text { Outpatients } \\
\text { receiving oral } \\
\text { chemotherapy }\end{array}$ & $\begin{array}{l}\text { Oncology } \\
\text { patients }\end{array}$ & Switzerland \\
\hline $\begin{array}{l}\text { Goldsmith } \\
\text { et al., } 2017\end{array}$ & $\begin{array}{l}\text { Understanding } \\
\text { the patient } \\
\text { experience of } \\
\text { cost-related } \\
\text { non-adherence } \\
\text { to prescription } \\
\text { medications } \\
\text { through } \\
\text { typology } \\
\text { development } \\
\text { and application }\end{array}$ & $\begin{array}{l}\text { Individual } \\
\text { interviews }\end{array}$ & $\begin{array}{l}\text { In which } \\
\text { situation } \\
\text { patients make } \\
\text { the decision not } \\
\text { to purchase } \\
\text { medicines }\end{array}$ & $\begin{array}{l}\text { Outpatients } \\
\text { who have not } \\
\text { taken their } \\
\text { drugs as } \\
\text { prescribed } \\
\text { because of } \\
\text { cost-related } \\
\text { problems }\end{array}$ & $\begin{array}{l}\text { Patients who } \\
\text { have } \\
\text { experiences } \\
\text { cost-related- } \\
\text { non-adherence }\end{array}$ & Canada \\
\hline $\begin{array}{l}\text { Habte et al., } \\
\qquad 2017\end{array}$ & $\begin{array}{l}\text { Barriers and } \\
\text { facilitators to } \\
\text { adherence to } \\
\text { anti-diabetic } \\
\text { medications: } \\
\text { Ethiopian } \\
\text { patients' } \\
\text { perspectives }\end{array}$ & $\begin{array}{l}\text { Individual } \\
\text { interviews }\end{array}$ & $\begin{array}{l}\text { Patients' } \\
\text { anti-diabetic } \\
\text { medication- } \\
\text { taking } \\
\text { experiences }\end{array}$ & $\begin{array}{l}\text { Diabetes } 2 \\
\text { patients in } \\
\text { public } \\
\text { outpatient } \\
\text { clinics }\end{array}$ & DM2 & Ethiopia \\
\hline
\end{tabular}


Table A1. Cont.

\begin{tabular}{|c|c|c|c|c|c|c|}
\hline Study & Title & Study Design & Concept & Context & Illness & $\begin{array}{l}\text { Country in } \\
\text { Which the } \\
\text { Study } \\
\text { Conducted }\end{array}$ \\
\hline $\begin{array}{l}\text { Harrold et al., } \\
2010\end{array}$ & $\begin{array}{l}\text { Patients and } \\
\text { providers view } \\
\text { gout differently: } \\
\text { a qualitative } \\
\text { study }\end{array}$ & $\begin{array}{l}\text { Individual } \\
\text { interviews }\end{array}$ & $\begin{array}{c}\text { Gout patients } \\
\text { views to ULD } \\
\text { (urate lowering } \\
\text { drugs) }\end{array}$ & $\begin{array}{l}\text { Gout patients' } \\
\text { adherence to } \\
\text { long-term urate } \\
\text { lowering } \\
\text { medication }\end{array}$ & Gout & United States \\
\hline $\begin{array}{l}\text { Hayden et al., } \\
2015\end{array}$ & $\begin{array}{c}\text { Patients' } \\
\text { adherence- } \\
\text { related beliefs } \\
\text { about } \\
\text { methotrexate: a } \\
\text { qualitative } \\
\text { study of the } \\
\text { role of written } \\
\text { patient } \\
\text { information }\end{array}$ & $\begin{array}{l}\text { Individual } \\
\text { interviews }\end{array}$ & $\begin{array}{l}\text { How patients' } \\
\text { beliefs and } \\
\text { concerns about } \\
\text { methotrexate } \\
\text { affected their } \\
\text { medicine taking }\end{array}$ & $\begin{array}{c}\text { Patients' } \\
\text { decisions about } \\
\text { taking } \\
\text { methotrexate in } \\
\text { outpatients } \\
\text { setting }\end{array}$ & $\begin{array}{l}\text { Inflammatory } \\
\text { arthritis }\end{array}$ & UK \\
\hline $\begin{array}{l}\text { Hedenrud et al., } \\
2019\end{array}$ & $\begin{array}{l}\text { "I did not know } \\
\text { it was so } \\
\text { important to } \\
\text { take it the } \\
\text { whole time"-- } \\
\text { self-reported } \\
\text { barriers to } \\
\text { medical } \\
\text { treatment } \\
\text { among } \\
\text { individuals } \\
\text { with asthma }\end{array}$ & $\begin{array}{l}\text { Individual } \\
\text { interviews }\end{array}$ & $\begin{array}{l}\text { Explore } \\
\text { self-perceived } \\
\text { barriers to } \\
\text { medication } \\
\text { adherence }\end{array}$ & $\begin{array}{l}\text { Outpatient } \\
\text { setting }\end{array}$ & Asthma & Sweden \\
\hline Ho et al., 2017 & $\begin{array}{l}\text { Barriers and } \\
\text { facilitators of } \\
\text { adherence to } \\
\text { antidepressants } \\
\text { among } \\
\text { outpatients } \\
\text { with major } \\
\text { depressive } \\
\text { disorder: A } \\
\text { qualitative } \\
\text { study }\end{array}$ & $\begin{array}{l}\text { Individual } \\
\text { interviews }\end{array}$ & $\begin{array}{c}\text { Patients' } \\
\text { non-adherence } \\
\text { in depression } \\
\text { medication }\end{array}$ & $\begin{array}{l}\text { Psychiatric } \\
\text { clinic in } \\
\text { government- } \\
\text { run hospital in } \\
\text { Malaysia }\end{array}$ & Depression & Malaysia \\
\hline $\begin{array}{c}\text { Hogan et al., } \\
2014\end{array}$ & $\begin{array}{c}\text { Factors } \\
\text { affecting } \\
\text { nebulised } \\
\text { medicine } \\
\text { adherence in } \\
\text { adult patients } \\
\text { with cystic } \\
\text { fibrosis: a } \\
\text { qualitative } \\
\text { study }\end{array}$ & $\begin{array}{l}\text { Individual } \\
\text { interviews }\end{array}$ & $\begin{array}{l}\text { Barriers and } \\
\text { facilitators of } \\
\text { nebulised } \\
\text { medicines used } \\
\text { for cystic } \\
\text { fibrosis }\end{array}$ & $\begin{array}{l}\text { Patients } \\
\text { recruited } \\
\text { through a } \\
\text { patient } \\
\text { organisation }\end{array}$ & Cystic fibrosis & Australia \\
\hline
\end{tabular}


Table A1. Cont.

\begin{tabular}{|c|c|c|c|c|c|c|}
\hline Study & Title & Study Design & Concept & Context & Illness & $\begin{array}{l}\text { Country in } \\
\text { Which the } \\
\text { Study } \\
\text { Conducted }\end{array}$ \\
\hline $\begin{array}{l}\text { Holtzman et al., } \\
\qquad 2015\end{array}$ & $\begin{array}{c}\text { Mapping } \\
\text { patient- } \\
\text { identified } \\
\text { barriers and } \\
\text { facilitators to } \\
\text { retention in } \\
\text { HIV care and } \\
\text { antiretroviral } \\
\text { therapy } \\
\text { adherence to } \\
\text { Andersen's } \\
\text { behavioural } \\
\text { model }\end{array}$ & $\begin{array}{l}\text { Individual } \\
\text { interviews }\end{array}$ & $\begin{array}{l}\text { HIV medication } \\
\text { adherence } \\
\text { linked to ABM } \\
\text { model }\end{array}$ & $\begin{array}{l}\text { HIV clinics in } \\
\text { Philadelphia, } \\
\text { USA }\end{array}$ & HIV / AIDS & United States \\
\hline $\begin{array}{l}\text { Huang et al., } \\
2020\end{array}$ & $\begin{array}{l}\text { “Why Am I Not } \\
\text { Taking } \\
\text { Medications?” } \\
\text { Barriers and } \\
\text { Facilitators of } \\
\text { Diabetes } \\
\text { Medication } \\
\text { Adherence } \\
\text { Across Different } \\
\text { Health Literacy } \\
\text { Levels }\end{array}$ & $\begin{array}{l}\text { Individual } \\
\text { interviews }\end{array}$ & $\begin{array}{l}\text { To explore } \\
\text { patients' } \\
\text { perceptions of } \\
\text { the barriers to } \\
\text { and facilitators } \\
\text { of medication } \\
\text { adherence } \\
\text { across different } \\
\text { levels of health } \\
\text { literacy }\end{array}$ & $\begin{array}{l}\text { how } \\
\text { individuals } \\
\text { make decisions } \\
\text { within an actual } \\
\text { real-world } \\
\text { situation }\end{array}$ & DM2 & United States \\
\hline $\begin{array}{l}\text { Iacorossi et al., } \\
2019\end{array}$ & $\begin{array}{l}\text { Qualitative } \\
\text { study of } \\
\text { patients with } \\
\text { metastatic } \\
\text { prostate cancer } \\
\text { to adherence of } \\
\text { hormone } \\
\text { therapy }\end{array}$ & $\begin{array}{l}\text { Individual } \\
\text { interviews }\end{array}$ & $\begin{array}{l}\text { Adherence to } \\
\text { oral hormone } \\
\text { treatment }\end{array}$ & $\begin{array}{l}\text { Outpatient } \\
\text { setting }\end{array}$ & Cancer & Italy \\
\hline $\begin{array}{l}\text { Jaffray et al., } \\
2014\end{array}$ & $\begin{array}{l}\text { Why do } \\
\text { patients } \\
\text { discontinue } \\
\text { antidepressant } \\
\text { therapy early? } \\
\text { A qualitative } \\
\text { study }\end{array}$ & $\begin{array}{l}\text { Individual } \\
\text { interviews }\end{array}$ & $\begin{array}{l}\text { Factors that } \\
\text { hinder or } \\
\text { facilitate the } \\
\text { continuation of } \\
\text { AD therapy }\end{array}$ & $\begin{array}{l}\text { Patients treated } \\
\text { in general } \\
\text { practice in } \\
\text { Scotland }\end{array}$ & Depression & UK \\
\hline Jarab et al., 2018 & $\begin{array}{l}\text { A focus group } \\
\text { study of } \\
\text { patient's } \\
\text { perspective and } \\
\text { experiences of } \\
\text { type } 2 \text { diabetes } \\
\text { and its } \\
\text { management in } \\
\text { Jordan }\end{array}$ & Focus group & $\begin{array}{l}\text { Non-adherence } \\
\text { to medication } \\
\text { as major barrier } \\
\text { to achieve good } \\
\text { results in } \\
\text { diabetes care }\end{array}$ & $\begin{array}{l}\text { Hospital } \\
\text { outpatients in } \\
\text { Jordan }\end{array}$ & DM2 & Jordan \\
\hline
\end{tabular}


Table A1. Cont.

\begin{tabular}{|c|c|c|c|c|c|c|}
\hline Study & Title & Study Design & Concept & Context & Illness & $\begin{array}{l}\text { Country in } \\
\text { Which the } \\
\text { Study } \\
\text { Conducted }\end{array}$ \\
\hline $\begin{array}{l}\text { Jeragh- } \\
\text { Alhaddad et al., } \\
2015\end{array}$ & $\begin{array}{l}\text { Barriers to } \\
\text { medication } \\
\text { taking among } \\
\text { Kuwaiti } \\
\text { patients with } \\
\text { type } 2 \text { diabetes: } \\
\text { A qualitative } \\
\text { study }\end{array}$ & $\begin{array}{l}\text { Individual } \\
\text { interviews }\end{array}$ & $\begin{array}{l}\text { Non-adherence } \\
\text { to medication } \\
\text { remains an } \\
\text { unresolved } \\
\text { problem }\end{array}$ & $\begin{array}{c}\text { Type } 2 \text { DM } \\
\text { patients from } \\
\text { general practice } \\
\text { and hospitals }\end{array}$ & DM2 & Kuwait \\
\hline Ju et al., 2018 & $\begin{array}{l}\text { Patient beliefs } \\
\text { and attitudes to } \\
\text { taking statins: } \\
\text { Systematic } \\
\text { review of } \\
\text { qualitative } \\
\text { studies }\end{array}$ & $\begin{array}{l}\text { Systematic } \\
\text { review }\end{array}$ & $\begin{array}{l}\text { Patients' } \\
\text { perspective on } \\
\text { statins in } \\
\text { primary or } \\
\text { secondary } \\
\text { prevention of } \\
\text { CVD }\end{array}$ & $\begin{array}{l}\text { Systematic } \\
\text { review on } \\
\text { qualitative } \\
\text { studies on } \\
\text { patients with } \\
\text { statins }\end{array}$ & $\begin{array}{c}\text { Cardiovascular } \\
\text { disease }\end{array}$ & Australia \\
\hline $\begin{array}{l}\text { Kassavou et al., } \\
2017\end{array}$ & $\begin{array}{l}\text { Reasons for } \\
\text { non-adherence } \\
\text { to car- } \\
\text { diometabolic } \\
\text { medications, } \\
\text { and } \\
\text { acceptability of } \\
\text { an interactive } \\
\text { voice response } \\
\text { intervention in } \\
\text { patients with } \\
\text { hypertension } \\
\text { and type } 2 \\
\text { diabetes in } \\
\text { primary care: a } \\
\text { qualitative } \\
\text { study }\end{array}$ & $\begin{array}{l}\text { Individual } \\
\text { interviews }\end{array}$ & $\begin{array}{c}\text { Patients' } \\
\text { non-adherence } \\
\text { to cardio } \\
\text { metabolic } \\
\text { medications }\end{array}$ & $\begin{array}{c}\text { General } \\
\text { practice } \\
\text { patients in the } \\
\text { UK }\end{array}$ & $\begin{array}{l}\mathrm{DM} 2 \text { and/or } \\
\text { hypertension }\end{array}$ & UK \\
\hline Kelly et al., 2014 & $\begin{array}{l}\text { Knowledge, } \\
\text { attitudes, and } \\
\text { beliefs of } \\
\text { patients and } \\
\text { carersregarding } \\
\text { medication } \\
\text { adherence: a } \\
\text { review of } \\
\text { qualitative } \\
\text { literature }\end{array}$ & $\begin{array}{l}\text { Systematic } \\
\text { review }\end{array}$ & $\begin{array}{l}\text { Systematic } \\
\text { qualitative } \\
\text { review on } \\
\text { factors which } \\
\text { can affect } \\
\text { medication } \\
\text { adherence }\end{array}$ & $\begin{array}{l}\text { Outpatients } \\
\text { setting }\end{array}$ & $\begin{array}{l}\text { Not mentioned } \\
\text { (patients in } \\
\text { general) }\end{array}$ & Ireland \\
\hline
\end{tabular}


Table A1. Cont.

\begin{tabular}{|c|c|c|c|c|c|c|}
\hline Study & Title & Study Design & Concept & Context & Illness & $\begin{array}{l}\text { Country in } \\
\text { Which the } \\
\text { Study } \\
\text { Conducted }\end{array}$ \\
\hline Kelly et al., 2018 & $\begin{array}{c}\text { Patients' } \\
\text { Attitudes and } \\
\text { Experiences of } \\
\text { Disease- } \\
\text { Modifying } \\
\text { Antirheumatic } \\
\text { Drugs in } \\
\text { Rheumatoid } \\
\text { Arthritis and } \\
\text { Spondylarthri- } \\
\text { tis: A } \\
\text { Qualitative } \\
\text { Synthesis }\end{array}$ & $\begin{array}{l}\text { Systematic } \\
\text { review }\end{array}$ & $\begin{array}{c}\text { Non-adherence } \\
\text { to } \\
\text { antirheumatic } \\
\text { drugs }\end{array}$ & $\begin{array}{l}\text { Qualitative } \\
\text { studies on } \\
\text { adherence to } \\
\text { RA drugs }\end{array}$ & $\begin{array}{l}\text { Rheumatoid } \\
\text { arthritis and } \\
\text { spondylarthri- } \\
\text { tis }\end{array}$ & Australia \\
\hline $\begin{array}{l}\text { King-Shier } \\
\text { et al., } 2017\end{array}$ & $\begin{array}{c}\text { Ethno-Cultural } \\
\text { Considerations } \\
\text { in Cardiac } \\
\text { Patients' } \\
\text { Medication } \\
\text { Adherence }\end{array}$ & $\begin{array}{l}\text { Individual } \\
\text { interviews }\end{array}$ & $\begin{array}{l}\text { How patients } \\
\text { manage to take } \\
\text { their cardiac } \\
\text { medication }\end{array}$ & $\begin{array}{l}\text { Outpatient } \\
\text { setting }\end{array}$ & $\begin{array}{c}\text { Cardiovascular } \\
\text { disease }\end{array}$ & Canada \\
\hline $\begin{array}{l}\text { Kobue et al., } \\
2017\end{array}$ & $\begin{array}{l}\text { "It's so hard } \\
\text { taking pills } \\
\text { when you don't } \\
\text { know what } \\
\text { they're for": a } \\
\text { qualitative } \\
\text { study of } \\
\text { patients' } \\
\text { medicine taking } \\
\text { behaviours and } \\
\text { conceptualisa- } \\
\text { tion of } \\
\text { medicines in } \\
\text { the context of } \\
\text { rheumatoid } \\
\text { arthritis }\end{array}$ & $\begin{array}{l}\text { Individual } \\
\text { interviews }\end{array}$ & $\begin{array}{l}\text { To understand } \\
\text { RA patients } \\
\text { medicine taking } \\
\text { behaviour }\end{array}$ & $\begin{array}{c}\text { Patients with } \\
\text { Rheumatoid } \\
\text { Arthritis (RA) } \\
\text { living in South } \\
\text { Africa }\end{array}$ & $\begin{array}{l}\text { Rheumatoid } \\
\text { arthritis }\end{array}$ & South Africa \\
\hline Koh et al., 2018 & $\begin{array}{l}\text { Access and } \\
\text { adherence to } \\
\text { medications for } \\
\text { the primary } \\
\text { and secondary } \\
\text { prevention of } \\
\text { atherosclerotic } \\
\text { cardiovascular } \\
\text { disease in } \\
\text { Singapore: a } \\
\text { qualitative } \\
\text { study }\end{array}$ & $\begin{array}{l}\text { Individual } \\
\text { interviews }\end{array}$ & $\begin{array}{l}\text { To understand } \\
\text { factors } \\
\text { influencing } \\
\text { patients' } \\
\text { adherence to } \\
\text { treatment }\end{array}$ & $\begin{array}{c}\text { Factors } \\
\text { impacting on } \\
\text { adherence with } \\
\text { cardiovascular } \\
\text { patients }\end{array}$ & $\begin{array}{c}\text { Cardiovascular } \\
\text { disease }\end{array}$ & Singapore \\
\hline
\end{tabular}


Table A1. Cont.

\begin{tabular}{|c|c|c|c|c|c|c|}
\hline Study & Title & Study Design & Concept & Context & Illness & $\begin{array}{l}\text { Country in } \\
\text { Which the } \\
\text { Study } \\
\text { Conducted }\end{array}$ \\
\hline $\begin{array}{c}\text { Kucukarslan } \\
\text { et al., } 2012\end{array}$ & $\begin{array}{c}\text { Exploring } \\
\text { patient } \\
\text { experiences } \\
\text { with } \\
\text { prescription } \\
\text { medicines to } \\
\text { identify unmet } \\
\text { patient needs: } \\
\text { Implications for } \\
\text { research and } \\
\text { practice }\end{array}$ & Focus group & $\begin{array}{c}\text { Patients' unmet } \\
\text { needs when } \\
\text { taking } \\
\text { prescribed } \\
\text { medicines }\end{array}$ & $\begin{array}{l}\text { Outpatients in } \\
\text { the US }\end{array}$ & $\begin{array}{c}\text { Cardiovascular } \\
\text { disease }\end{array}$ & United States \\
\hline $\begin{array}{l}\text { Lambert- } \\
\text { Kerzner et al., } \\
2015\end{array}$ & $\begin{array}{l}\text { Perspectives of } \\
\text { patients on } \\
\text { factors relating } \\
\text { to adherence to } \\
\text { post-acute } \\
\text { coronary } \\
\text { syndrome } \\
\text { medical } \\
\text { regimens }\end{array}$ & $\begin{array}{l}\text { Individual } \\
\text { interviews }\end{array}$ & $\begin{array}{l}\text { Adherence to } \\
\text { cardio } \\
\text { protective } \\
\text { medications } \\
\text { after acute } \\
\text { coronary } \\
\text { syndrome }\end{array}$ & $\begin{array}{c}\text { Patients } \\
\text { attending an } \\
\text { RCT on } \\
\text { multi-faceted } \\
\text { intervention to } \\
\text { improve cardiac } \\
\text { medication } \\
\text { adherence }\end{array}$ & $\begin{array}{c}\text { Cardiovascular } \\
\text { disease }\end{array}$ & United States \\
\hline $\begin{array}{l}\text { Lyimo et al., } \\
2012\end{array}$ & $\begin{array}{c}\text { Determinants } \\
\text { of antiretroviral } \\
\text { therapy } \\
\text { adherence in } \\
\text { northern } \\
\text { Tanzania: a } \\
\text { comprehensive } \\
\text { picture from the } \\
\text { patient } \\
\text { perspective }\end{array}$ & $\begin{array}{l}\text { Individual } \\
\text { interviews }\end{array}$ & $\begin{array}{l}\text { Understanding } \\
\text { of barriers and } \\
\text { facilitators of } \\
\text { antiretroviral } \\
\text { therapy }\end{array}$ & $\begin{array}{l}\text { Health centres } \\
\text { in Tanzania }\end{array}$ & HIV / AIDS & Tanzania \\
\hline $\begin{array}{l}\text { Maffoni et al., } \\
2020\end{array}$ & $\begin{array}{l}\text { Medication } \\
\text { adherence in } \\
\text { the older adults } \\
\text { with chronic } \\
\text { multimorbidity: } \\
\text { a systematic } \\
\text { review of } \\
\text { qualitative } \\
\text { studies on } \\
\text { patient's } \\
\text { experience. } \\
\text { [Review] }\end{array}$ & $\begin{array}{l}\text { Systematic } \\
\text { review }\end{array}$ & $\begin{array}{l}\text { Older patient's } \\
\text { perspective on } \\
\text { medication } \\
\text { adherence }\end{array}$ & $\begin{array}{c}\text { medication } \\
\text { adherence in } \\
\text { chronic diseases }\end{array}$ & $\begin{array}{l}\text { Other: } \\
\text { hypertension, } \\
\text { heart disease, } \\
\text { COPD, asthma }\end{array}$ & $\begin{array}{c}\text { Italy, Portugal, } \\
\text { and Poland }\end{array}$ \\
\hline $\begin{array}{l}\text { Marshall et al., } \\
2012\end{array}$ & $\begin{array}{c}\text { Lay } \\
\text { perspectives on } \\
\text { hypertension } \\
\text { and drug } \\
\text { adherence: } \\
\text { systematic } \\
\text { review of } \\
\text { qualitative } \\
\text { research }\end{array}$ & $\begin{array}{l}\text { Systematic } \\
\text { review }\end{array}$ & $\begin{array}{c}\text { To better } \\
\text { understand } \\
\text { patients' } \\
\text { perspectives to } \\
\text { medication } \\
\text { adherence }\end{array}$ & $\begin{array}{l}\text { Qualitative } \\
\text { studies on } \\
\text { patients using } \\
\text { antihyperten- } \\
\text { sive } \\
\text { drugs }\end{array}$ & $\begin{array}{c}\text { Cardiovascular } \\
\text { disease }\end{array}$ & UK \\
\hline
\end{tabular}


Table A1. Cont.

\begin{tabular}{|c|c|c|c|c|c|c|}
\hline Study & Title & Study Design & Concept & Context & Illness & $\begin{array}{l}\text { Country in } \\
\text { Which the } \\
\text { Study } \\
\text { Conducted }\end{array}$ \\
\hline $\begin{array}{l}\text { McDonald } \\
\text { et al., } 2019\end{array}$ & $\begin{array}{c}\text { A } \\
\text { theory-driven } \\
\text { qualitative } \\
\text { study exploring } \\
\text { issues relating } \\
\text { to adherence to } \\
\text { topical } \\
\text { glaucoma } \\
\text { medications }\end{array}$ & $\begin{array}{l}\text { Individual } \\
\text { interviews }\end{array}$ & $\begin{array}{l}\text { Investigating } \\
\text { patients' } \\
\text { perceptions of } \\
\text { their illness }\end{array}$ & $\begin{array}{l}\text { Two outpatient } \\
\text { glaucoma } \\
\text { clinics }\end{array}$ & Glaucoma & UK \\
\hline $\begin{array}{l}\text { McKillop et al., } \\
2013\end{array}$ & $\begin{array}{c}\text { Patients' } \\
\text { experience and } \\
\text { perceptions of } \\
\text { polypharmacy } \\
\text { in chronic } \\
\text { kidney disease } \\
\text { and its impact } \\
\text { on adherent } \\
\text { behaviour }\end{array}$ & $\begin{array}{l}\text { Individual } \\
\text { interviews }\end{array}$ & $\begin{array}{l}\text { Polypharmacy } \\
\text { is common in } \\
\text { chronic kidney } \\
\text { disease and } \\
\text { associated with } \\
\text { medication } \\
\text { adherence }\end{array}$ & $\begin{array}{l}\text { Patients at a } \\
\text { nephrology } \\
\text { clinic }\end{array}$ & $\begin{array}{c}\text { Chronic kidney } \\
\text { disease }\end{array}$ & UK \\
\hline $\begin{array}{l}\text { McSharry et al., } \\
2016\end{array}$ & $\begin{array}{l}\text { Systematic } \\
\text { Review or } \\
\text { Meta-analysis } \\
\text { Perceptions and } \\
\text { experiences of } \\
\text { taking oral } \\
\text { medications for } \\
\text { the treatment of } \\
\text { Type } 2 \text { diabetes } \\
\text { mellitus: a } \\
\text { systematic } \\
\text { review and } \\
\text { meta-synthesis } \\
\text { of qualitative } \\
\text { studies }\end{array}$ & $\begin{array}{l}\text { Systematic } \\
\text { review }\end{array}$ & $\begin{array}{l}\text { DM2 patients' } \\
\text { adherence to } \\
\text { diabetes } \\
\text { medicines }\end{array}$ & $\begin{array}{l}\text { Outpatients } \\
\text { setting }\end{array}$ & DM2 & UK \\
\hline $\begin{array}{l}\text { Meraz et al., } \\
2020\end{array}$ & $\begin{array}{c}\text { Medication } \\
\text { Non-adherence } \\
\text { or Self-care? } \\
\text { Understanding } \\
\text { the Medication } \\
\text { Decision- } \\
\text { Making Process } \\
\text { and } \\
\text { Experiences of } \\
\text { Older Adults } \\
\text { With Heart } \\
\text { Failure }\end{array}$ & $\begin{array}{l}\text { Individual } \\
\text { interviews }\end{array}$ & $\begin{array}{l}\text { Understanding } \\
\text { the medicine } \\
\text { decision- } \\
\text { making } \\
\text { process }\end{array}$ & $\begin{array}{l}\text { Community } \\
\text { setting }\end{array}$ & $\begin{array}{c}\text { Cardiovascular } \\
\text { disease }\end{array}$ & United States \\
\hline
\end{tabular}


Table A1. Cont.

\begin{tabular}{|c|c|c|c|c|c|c|}
\hline Study & Title & Study Design & Concept & Context & Illness & $\begin{array}{l}\text { Country in } \\
\text { Which the } \\
\text { Study } \\
\text { Conducted }\end{array}$ \\
\hline $\begin{array}{l}\text { Miller et al., } \\
2010\end{array}$ & $\begin{array}{c}\text { Why are } \\
\text { antiretroviral } \\
\text { treatment } \\
\text { patients lost to } \\
\text { follow-up? A } \\
\text { qualitative } \\
\text { study from } \\
\text { South Africa }\end{array}$ & $\begin{array}{l}\text { Individual } \\
\text { interviews }\end{array}$ & $\begin{array}{c}\text { Reasons for } \\
\text { non-adherence }\end{array}$ & $\begin{array}{c}\text { HIV / AIDS } \\
\text { patients } \\
\text { receiving ART }\end{array}$ & HIV / AIDS & South Africa \\
\hline $\begin{array}{c}\text { Ming et al., } \\
2011\end{array}$ & $\begin{array}{l}\text { Perspectives of } \\
\text { heart failure } \\
\text { patients in } \\
\text { Malaysia } \\
\text { towards } \\
\text { medications } \\
\text { and disease } \\
\text { state } \\
\text { management: } \\
\text { Findings from a } \\
\text { qualitative } \\
\text { study }\end{array}$ & $\begin{array}{l}\text { Individual } \\
\text { interviews }\end{array}$ & $\begin{array}{c}\text { Patient } \\
\text { perspectives in } \\
\text { the } \\
\text { management of } \\
\text { heart failure }\end{array}$ & $\begin{array}{l}\text { General } \\
\text { hospital, } \\
\text { Malaysia }\end{array}$ & $\begin{array}{c}\text { Cardiovascular } \\
\text { disease }\end{array}$ & Malaysia \\
\hline $\begin{array}{c}\text { Mostafavi et al., } \\
2021\end{array}$ & $\begin{array}{c}\text { The } \\
\text { psychosocial } \\
\text { barriers to } \\
\text { medication } \\
\text { adherence of } \\
\text { patients with } \\
\text { type } 2 \text { diabetes: } \\
\text { a qualitative } \\
\text { study }\end{array}$ & $\begin{array}{l}\text { Individual } \\
\text { interviews }\end{array}$ & $\begin{array}{l}\text { Barriers to } \\
\text { medication } \\
\text { adherence }\end{array}$ & $\begin{array}{l}\text { Outpatient } \\
\text { setting }\end{array}$ & DM2 & Iran \\
\hline $\begin{array}{l}\text { Muiruri et al., } \\
2020\end{array}$ & $\begin{array}{l}\text { Why do people } \\
\text { living with HIV } \\
\text { adhere to } \\
\text { antiretroviral } \\
\text { therapy and not } \\
\text { comorbid } \\
\text { cardiovascular } \\
\text { disease } \\
\text { medications? A } \\
\text { qualitative } \\
\text { inquiry }\end{array}$ & $\begin{array}{l}\text { Focus groups } \\
\text { and individual } \\
\text { interviews }\end{array}$ & $\begin{array}{l}\text { HIV-patients } \\
\text { adherence to } \\
\text { cardiovascular } \\
\text { medications }\end{array}$ & $\begin{array}{l}\text { Outpatient } \\
\text { setting }\end{array}$ & $\begin{array}{c}\text { Cardiovascular } \\
\text { disease }\end{array}$ & United States \\
\hline $\begin{array}{l}\text { Nielsen et al., } \\
2018\end{array}$ & $\begin{array}{l}\text { Adherence to } \\
\text { medication in } \\
\text { patients with } \\
\text { chronic kidney } \\
\text { disease: a } \\
\text { systematic } \\
\text { review of } \\
\text { qualitative } \\
\text { research }\end{array}$ & $\begin{array}{l}\text { Systematic } \\
\text { review }\end{array}$ & $\begin{array}{l}\text { Non-adherence } \\
\text { to multipharma- } \\
\text { cological } \\
\text { treatment }\end{array}$ & $\begin{array}{l}\text { Nephrology } \\
\text { unit in } \\
\text { Denmark }\end{array}$ & $\begin{array}{c}\text { Chronic kidney } \\
\text { disease }\end{array}$ & Denmark \\
\hline
\end{tabular}


Table A1. Cont.

\begin{tabular}{|c|c|c|c|c|c|c|}
\hline Study & Title & Study Design & Concept & Context & Illness & $\begin{array}{l}\text { Country in } \\
\text { Which the } \\
\text { Study } \\
\text { Conducted }\end{array}$ \\
\hline $\begin{array}{l}\text { Oshotse et al., } \\
2018\end{array}$ & $\begin{array}{l}\text { Self-Efficacy } \\
\text { and Adherence } \\
\text { Behaviours in } \\
\text { Rheumatoid } \\
\text { Arthritis } \\
\text { Patients }\end{array}$ & $\begin{array}{l}\text { Other: Focus } \\
\text { group and } \\
\text { individual } \\
\text { interviews }\end{array}$ & $\begin{array}{c}\text { How } \\
\text { self-efficacy and } \\
\text { adherence is } \\
\text { influencing } \\
\text { medication } \\
\text { taking }\end{array}$ & $\begin{array}{l}\text { RA patients' } \\
\text { self- } \\
\text { management }\end{array}$ & $\begin{array}{l}\text { Rheumatoid } \\
\text { arthritis }\end{array}$ & United States \\
\hline $\begin{array}{c}\text { Pagès- } \\
\text { Puigdemont } \\
\text { et al., } 2016\end{array}$ & $\begin{array}{c}\text { Patients' } \\
\text { Perspective of } \\
\text { Medication } \\
\text { Adherence in } \\
\text { Chronic } \\
\text { Conditions: A } \\
\text { Qualitative } \\
\text { Study }\end{array}$ & Focus group & $\begin{array}{l}\text { Medication } \\
\text { adherence in } \\
\text { chronic } \\
\text { conditions }\end{array}$ & $\begin{array}{c}\text { Patents' } \\
\text { perspectives in } \\
\text { medication } \\
\text { management in } \\
\text { chronic diseases }\end{array}$ & $\begin{array}{l}\text { Chronic } \\
\text { diseases }\end{array}$ & Spain \\
\hline Patel et al., 2015 & $\begin{array}{l}\text { Concerns and } \\
\text { perceptions } \\
\text { about necessity } \\
\text { in relation to } \\
\text { insulin therapy } \\
\text { in an ethnically } \\
\text { diverse UK } \\
\text { population with } \\
\text { type } 2 \text { diabetes: } \\
\text { a qualitative } \\
\text { study focusing } \\
\text { mainly on } \\
\text { people of South } \\
\text { Asian origin }\end{array}$ & $\begin{array}{l}\text { Individual } \\
\text { interviews }\end{array}$ & $\begin{array}{l}\text { Accepting } \\
\text { insulin as } \\
\text { medication to } \\
\text { DM2 }\end{array}$ & $\begin{array}{l}\text { Ethnic } \\
\text { population } \\
\text { living in UK } \\
\text { with DM2 and } \\
\text { their adherence } \\
\text { to insulin }\end{array}$ & DM2 & UK \\
\hline $\begin{array}{l}\text { Peláez et al., } \\
2016\end{array}$ & $\begin{array}{c}\text { How can } \\
\text { adherence to } \\
\text { asthma } \\
\text { medication } \\
\text { been enhanced? } \\
\text { Triangulation of } \\
\text { key asthma } \\
\text { stakeholders' } \\
\text { perspectives }\end{array}$ & Focus group & $\begin{array}{c}\text { Asthma } \\
\text { patients' } \\
\text { adherence to } \\
\text { medications }\end{array}$ & $\begin{array}{c}\text { To explore } \\
\text { interventions } \\
\text { which enhances } \\
\text { adherence to } \\
\text { asthma } \\
\text { medication in } \\
\text { long-term }\end{array}$ & Asthma & Canada \\
\hline $\begin{array}{l}\text { Pettersen et al., } \\
2018\end{array}$ & $\begin{array}{l}\text { Challenges } \\
\text { adhering to a } \\
\text { medication } \\
\text { regimen } \\
\text { following } \\
\text { first-time } \\
\text { percutaneous } \\
\text { coronary } \\
\text { intervention: A } \\
\text { patient } \\
\text { perspective }\end{array}$ & $\begin{array}{l}\text { Individual } \\
\text { interviews }\end{array}$ & $\begin{array}{l}\text { Adherence to } \\
\text { cardiovascular } \\
\text { medication } \\
\text { after } \\
\text { percutaneous } \\
\text { coronary } \\
\text { intervention }\end{array}$ & $\begin{array}{l}\text { Cardiology unit } \\
\text { in Norway }\end{array}$ & $\begin{array}{c}\text { Cardiovascular } \\
\text { disease }\end{array}$ & Norway \\
\hline
\end{tabular}


Table A1. Cont.

\begin{tabular}{|c|c|c|c|c|c|c|}
\hline Study & Title & Study Design & Concept & Context & Illness & $\begin{array}{l}\text { Country in } \\
\text { Which the } \\
\text { Study } \\
\text { Conducted }\end{array}$ \\
\hline $\begin{array}{l}\text { Polinski et al., } \\
2014\end{array}$ & $\begin{array}{l}\text { A matter of } \\
\text { trust: patient } \\
\text { barriers to } \\
\text { primary } \\
\text { medication } \\
\text { adherence }\end{array}$ & Focus group & $\begin{array}{c}\text { Patients' } \\
\text { adherence to an- } \\
\text { tihypertensive } \\
\text { medications } \\
\text { remains } \\
\text { suboptimal }\end{array}$ & $\begin{array}{l}\text { Patients who } \\
\text { did not pick up } \\
\text { the first antihy- } \\
\text { pertensive } \\
\text { prescription }\end{array}$ & $\begin{array}{l}\text { Cardiovascular } \\
\text { disease }\end{array}$ & United States \\
\hline $\begin{array}{l}\text { Rahmawati } \\
\text { et al., } 2018\end{array}$ & $\begin{array}{c}\text { Understanding } \\
\text { untreated } \\
\text { hypertension } \\
\text { from patients' } \\
\text { point of view: } \\
\text { A qualitative } \\
\text { study in rural } \\
\text { Yogyakarta } \\
\text { province, } \\
\text { Indonesia }\end{array}$ & $\begin{array}{l}\text { Individual } \\
\text { interviews }\end{array}$ & $\begin{array}{c}\text { To explore } \\
\text { perspectives } \\
\text { from patients } \\
\text { who do not take } \\
\text { anti- } \\
\text { hypertensive } \\
\text { medications }\end{array}$ & & $\begin{array}{l}\text { Cardiovascular } \\
\text { disease }\end{array}$ & Indonesia \\
\hline $\begin{array}{l}\text { Rashid et al., } \\
2014\end{array}$ & $\begin{array}{l}\text { Medication } \\
\text { taking in } \\
\text { coronary artery } \\
\text { disease: A } \\
\text { systematic } \\
\text { review and } \\
\text { qualitative } \\
\text { synthesis }\end{array}$ & $\begin{array}{l}\text { Systematic } \\
\text { review }\end{array}$ & $\begin{array}{c}\text { Patients' } \\
\text { discontinuation } \\
\text { to secondary } \\
\text { prevention } \\
\text { medication for } \\
\text { coronary artery } \\
\text { disease }\end{array}$ & $\begin{array}{l}\text { Qualitative } \\
\text { research about } \\
\text { the medication- } \\
\text { taking } \\
\text { experiences }\end{array}$ & $\begin{array}{l}\text { Cardiovascular } \\
\text { disease }\end{array}$ & UK \\
\hline $\begin{array}{l}\text { Rathbone et al., } \\
\qquad 2017\end{array}$ & $\begin{array}{l}\text { A systematic } \\
\text { review and } \\
\text { thematic } \\
\text { synthesis of } \\
\text { patients' } \\
\text { experience of } \\
\text { medicines } \\
\text { adherence }\end{array}$ & $\begin{array}{l}\text { Systematic } \\
\text { review }\end{array}$ & $\begin{array}{l}\text { Phenomenology } \\
\text { has a place in } \\
\text { studying } \\
\text { adherence }\end{array}$ & $\begin{array}{l}\text { Phenomenological } \\
\text { papers } \\
\text { studying } \\
\text { medication } \\
\text { adherence }\end{array}$ & $\begin{array}{l}\text { Cardiovascular } \\
\text { disease }\end{array}$ & UK \\
\hline $\begin{array}{l}\text { Rezaei et al., } \\
2019\end{array}$ & $\begin{array}{c}\text { Barriers of } \\
\text { medication } \\
\text { adherence in } \\
\text { patients with } \\
\text { type-2 diabetes: } \\
\text { A pilot } \\
\text { qualitative } \\
\text { study }\end{array}$ & $\begin{array}{l}\text { Individual } \\
\text { interviews }\end{array}$ & $\begin{array}{l}\text { Patients with } \\
\text { type } 2 \text { diabetes } \\
\text { have poor } \\
\text { adherence to } \\
\text { the therapeutic } \\
\text { regime }\end{array}$ & $\begin{array}{l}\text { Outpatient } \\
\text { setting }\end{array}$ & DM2 & Iran \\
\hline $\begin{array}{l}\text { Richardson } \\
\text { et al., } 2016\end{array}$ & $\begin{array}{l}\text { A joint effort } \\
\text { over a period of } \\
\text { time: Factors } \\
\text { affecting use of } \\
\text { urate-lowering } \\
\text { therapy for } \\
\text { long-Term } \\
\text { treatment of } \\
\text { gout }\end{array}$ & $\begin{array}{l}\text { Individual } \\
\text { interviews }\end{array}$ & $\begin{array}{l}\text { Reasons for } \\
\text { non-adherence } \\
\text { to gout } \\
\text { treatment }\end{array}$ & $\begin{array}{l}\text { GP patients } \\
\text { through the UK }\end{array}$ & Gout & UK \\
\hline
\end{tabular}


Table A1. Cont.

\begin{tabular}{|c|c|c|c|c|c|c|}
\hline Study & Title & Study Design & Concept & Context & Illness & $\begin{array}{l}\text { Country in } \\
\text { Which the } \\
\text { Study } \\
\text { Conducted }\end{array}$ \\
\hline $\begin{array}{l}\text { Rifkin et al., } \\
2010\end{array}$ & $\begin{array}{c}\text { Medication } \\
\text { adherence } \\
\text { behaviour and } \\
\text { priorities } \\
\text { among older } \\
\text { adults with } \\
\text { CKD: A } \\
\text { semistructured } \\
\text { interview study }\end{array}$ & $\begin{array}{l}\text { Individual } \\
\text { interviews }\end{array}$ & $\begin{array}{l}\text { How patients } \\
\text { with multiple } \\
\text { problems in } \\
\text { kidney disease } \\
\text { prioritise their } \\
\text { medications }\end{array}$ & $\begin{array}{l}\text { Community- } \\
\text { dwelling } \\
\text { patients with } \\
\text { kidney disease }\end{array}$ & $\begin{array}{c}\text { Chronic kidney } \\
\text { disease }\end{array}$ & United States \\
\hline $\begin{array}{c}\text { Rowell- } \\
\text { Cunsolo et al., } \\
2020\end{array}$ & $\begin{array}{l}\text { Barriers to } \\
\text { optimal } \\
\text { antiretroviral } \\
\text { therapy } \\
\text { adherence } \\
\text { among } \\
\text { HIV-infected } \\
\text { formerly } \\
\text { incarcerated } \\
\text { individuals in } \\
\text { New York City }\end{array}$ & $\begin{array}{l}\text { Individual } \\
\text { interviews }\end{array}$ & $\begin{array}{c}\text { Investigate } \\
\text { barriers to ART }\end{array}$ & $\begin{array}{l}\text { Outpatient } \\
\text { setting }\end{array}$ & HIV / AIDS & United States \\
\hline $\begin{array}{l}\text { Saleem et al., } \\
2012\end{array}$ & $\begin{array}{l}\text { Drug attitude } \\
\text { and adherence: } \\
\text { A qualitative } \\
\text { insight of } \\
\text { patients with } \\
\text { hypertension }\end{array}$ & $\begin{array}{l}\text { Individual } \\
\text { interviews }\end{array}$ & $\begin{array}{l}\text { Patients' insight } \\
\text { to hypertension } \\
\text { medication }\end{array}$ & $\begin{array}{l}\text { Outpatient } \\
\text { setting }\end{array}$ & $\begin{array}{c}\text { Cardiovascular } \\
\text { disease }\end{array}$ & Pakistan \\
\hline $\begin{array}{l}\text { Sapkota et al., } \\
2018\end{array}$ & $\begin{array}{c}\text { Nepalese } \\
\text { patients' } \\
\text { anti-diabetic } \\
\text { medication } \\
\text { taking } \\
\text { behaviour: an } \\
\text { exploratory } \\
\text { study }\end{array}$ & $\begin{array}{l}\text { Individual } \\
\text { interviews }\end{array}$ & $\begin{array}{l}\text { Diabetes causes } \\
\text { a huge burden } \\
\text { for low- and } \\
\text { middle-income } \\
\text { countries }\end{array}$ & $\begin{array}{l}\text { Nepalese type } 2 \\
\text { DM patients in } \\
\text { Nepal and } \\
\text { Australia }\end{array}$ & DM2 & $\begin{array}{l}\text { Nepal and } \\
\text { Australia }\end{array}$ \\
\hline $\begin{array}{c}\text { Schatz et al., } \\
2019\end{array}$ & $\begin{array}{l}\text { "For us here, } \\
\text { we remind } \\
\text { ourselves": } \\
\text { strategies and } \\
\text { barriers to ART } \\
\text { access and } \\
\text { adherence } \\
\text { among older } \\
\text { Ugandans }\end{array}$ & $\begin{array}{l}\text { Individual } \\
\text { interviews }\end{array}$ & $\begin{array}{c}\text { Antiretroviral } \\
\text { therapy among } \\
\text { older Africans }\end{array}$ & $\begin{array}{c}\text { Older adults in } \\
\text { Uganda with } \\
\text { HIV }\end{array}$ & HIV / AIDS & Uganda \\
\hline $\begin{array}{l}\text { Shalihu et al., } \\
2014\end{array}$ & $\begin{array}{c}\text { Namibian } \\
\text { prisoners } \\
\text { describe } \\
\text { barriers to HIV } \\
\text { antiretroviral } \\
\text { therapy } \\
\text { adherence }\end{array}$ & $\begin{array}{l}\text { Individual } \\
\text { interviews }\end{array}$ & $\begin{array}{l}\text { Adherence to } \\
\text { HIV medication }\end{array}$ & $\begin{array}{l}\text { Patients with } \\
\text { AIDS in a } \\
\text { Namibian } \\
\text { prison }\end{array}$ & HIV / AIDS & Namibia \\
\hline
\end{tabular}


Table A1. Cont.

\begin{tabular}{|c|c|c|c|c|c|c|}
\hline Study & Title & Study Design & Concept & Context & Illness & $\begin{array}{l}\text { Country in } \\
\text { Which the } \\
\text { Study } \\
\text { Conducted }\end{array}$ \\
\hline $\begin{array}{c}\text { Shaw et al., } \\
2018\end{array}$ & $\begin{array}{l}\text { Rheumatoid } \\
\text { arthritis } \\
\text { patients' } \\
\text { motivations for } \\
\text { accepting or } \\
\text { resisting } \\
\text { disease- } \\
\text { modifying } \\
\text { antirheumatic } \\
\text { drug treatment } \\
\text { regimens }\end{array}$ & $\begin{array}{l}\text { Individual } \\
\text { interviews }\end{array}$ & $\begin{array}{l}\text { Patients' } \\
\text { decision to } \\
\text { accept or resist } \\
\text { disease } \\
\text { modifying anti } \\
\text { rheumatic } \\
\text { drugs }\end{array}$ & $\begin{array}{c}\text { Four } \\
\text { rheumatology } \\
\text { clinics in } \\
\text { Pittsburgh }\end{array}$ & $\begin{array}{l}\text { Rheumatoid } \\
\text { arthritis }\end{array}$ & United States \\
\hline $\begin{array}{l}\text { Shiyanbola } \\
\text { et al., } 2018\end{array}$ & $\begin{array}{l}\text { "I did not want } \\
\text { to take that } \\
\text { medicine": } \\
\text { African- } \\
\text { Americans' } \\
\text { reasons for } \\
\text { diabetes } \\
\text { medication } \\
\text { non-adherence } \\
\text { and perceived } \\
\text { solutions for } \\
\text { enhancing } \\
\text { adherence }\end{array}$ & Focus group & $\begin{array}{l}\text { Diabetes is dis- } \\
\text { proportionally } \\
\text { burdensome } \\
\text { among African- } \\
\text { Americans } \\
\text { (AAs) and } \\
\text { medication } \\
\text { adherence is } \\
\text { important for } \\
\text { optimal } \\
\text { outcomes. }\end{array}$ & $\begin{array}{l}\text { Community } \\
\text { African } \\
\text { American type } \\
2 \text { DM patients }\end{array}$ & DM2 & United States \\
\hline $\begin{array}{l}\text { Souter et al., } \\
2014\end{array}$ & $\begin{array}{l}\text { Optimisation of } \\
\text { secondary } \\
\text { prevention of } \\
\text { stroke: A } \\
\text { qualitative } \\
\text { study of stroke } \\
\text { patients' beliefs, } \\
\text { concerns and } \\
\text { difficulties with } \\
\text { their medicines }\end{array}$ & $\begin{array}{l}\text { Individual } \\
\text { interviews }\end{array}$ & $\begin{array}{l}\text { Optimisation of } \\
\text { secondary } \\
\text { prevention of } \\
\text { stroke }\end{array}$ & $\begin{array}{c}\text { Patients } \\
\text { discharged } \\
\text { from stroke } \\
\text { rehabilitation }\end{array}$ & $\begin{array}{c}\text { Cardiovascular } \\
\text { disease }\end{array}$ & UK \\
\hline $\begin{array}{l}\text { Srimongkon } \\
\text { et al., } 2018\end{array}$ & $\begin{array}{l}\text { Consumer- } \\
\text { related factors } \\
\text { influencing } \\
\text { antidepressant } \\
\text { adherence in } \\
\text { unipolar } \\
\text { depression: a } \\
\text { qualitative } \\
\text { study }\end{array}$ & $\begin{array}{l}\text { Individual } \\
\text { interviews }\end{array}$ & $\begin{array}{c}\text { Adherence at } \\
\text { different stages: } \\
\text { initiation, im- } \\
\text { plementation, } \\
\text { and } \\
\text { discontinuation } \\
\text { of medication }\end{array}$ & $\begin{array}{l}\text { Outpatient } \\
\text { setting }\end{array}$ & Depression & Australia \\
\hline
\end{tabular}


Table A1. Cont.

\begin{tabular}{|c|c|c|c|c|c|c|}
\hline Study & Title & Study Design & Concept & Context & Illness & $\begin{array}{l}\text { Country in } \\
\text { Which the } \\
\text { Study } \\
\text { Conducted }\end{array}$ \\
\hline Stern et al., 2017 & $\begin{array}{l}\text { Conceptions of } \\
\text { agency and } \\
\text { constraint for } \\
\text { HIV-positive } \\
\text { patients and } \\
\text { healthcare } \\
\text { workers to } \\
\text { support } \\
\text { long-term } \\
\text { engagement } \\
\text { with } \\
\text { antiretroviral } \\
\text { therapy care in } \\
\text { Khayelitsha, } \\
\text { South Africa }\end{array}$ & $\begin{array}{l}\text { Individual } \\
\text { interviews }\end{array}$ & $\begin{array}{l}\text { Barriers to } \\
\text { long-term ART } \\
\text { adherence is } \\
\text { critical in HIV } \\
\text { management }\end{array}$ & $\begin{array}{c}\text { Three HIV } \\
\text { clinics in South } \\
\text { Africa }\end{array}$ & HIV / AIDS & South Africa \\
\hline $\begin{array}{l}\text { Stryker et al., } \\
2010\end{array}$ & $\begin{array}{l}\text { An Exploratory } \\
\text { Study of } \\
\text { Factors } \\
\text { Influencing } \\
\text { Glaucoma } \\
\text { Treatment } \\
\text { Adherence }\end{array}$ & $\begin{array}{l}\text { Individual } \\
\text { interviews }\end{array}$ & $\begin{array}{l}\text { Patient } \\
\text { adherence to } \\
\text { glaucoma } \\
\text { treatment } \\
\text { regimens is } \\
\text { often } \\
\text { suboptimal }\end{array}$ & $\begin{array}{l}\text { Veteran Affairs } \\
\text { hospital in US }\end{array}$ & Glaucoma & United States \\
\hline Tong et al., 2011 & $\begin{array}{l}\text { The } \\
\text { perspectives of } \\
\text { kidney } \\
\text { transplant } \\
\text { recipients on } \\
\text { medicine } \\
\text { taking: A } \\
\text { systematic } \\
\text { review of } \\
\text { qualitative } \\
\text { studies }\end{array}$ & $\begin{array}{l}\text { Systematic } \\
\text { review }\end{array}$ & $\begin{array}{l}\text { Non-adherence } \\
\text { to medication } \\
\text { regimens after } \\
\text { kidney } \\
\text { transplantation }\end{array}$ & $\begin{array}{l}\text { Qualitative } \\
\text { studies using } \\
\text { interviews, } \\
\text { focus groups, } \\
\text { document } \\
\text { analysis or } \\
\text { observations to } \\
\text { explore the } \\
\text { perspectives of } \\
\text { adult kidney } \\
\text { transplant } \\
\text { recipients }\end{array}$ & $\begin{array}{c}\text { Kidney } \\
\text { transplants }\end{array}$ & Australia \\
\hline $\begin{array}{l}\text { Tordoff et al, } \\
2010\end{array}$ & $\begin{array}{l}\text { “It's just } \\
\text { routine." A } \\
\text { qualitative } \\
\text { study of } \\
\text { medicine- } \\
\text { taking amongst } \\
\text { older people in } \\
\text { New Zealand }\end{array}$ & $\begin{array}{l}\text { Individual } \\
\text { interviews }\end{array}$ & $\begin{array}{l}\text { Many older } \\
\text { people use } \\
\text { large numbers } \\
\text { of medicines } \\
\text { and are more } \\
\text { likely to have } \\
\text { problems } \\
\text { taking them }\end{array}$ & $\begin{array}{c}\text { Community } \\
\text { patients in New } \\
\text { Zealand }\end{array}$ & $\begin{array}{l}\text { Not mentioned } \\
\text { (patients in } \\
\text { general) }\end{array}$ & New Zealand \\
\hline $\begin{array}{c}\text { Tranulis et al., } \\
2011\end{array}$ & $\begin{array}{l}\text { Becoming } \\
\text { adherent to } \\
\text { antipsychotics: } \\
\text { a qualitative } \\
\text { study of } \\
\text { treatment- } \\
\text { experienced } \\
\text { schizophrenia } \\
\text { patients }\end{array}$ & $\begin{array}{l}\text { Individual } \\
\text { interviews }\end{array}$ & $\begin{array}{c}\text { Patients' } \\
\text { perspectives on } \\
\text { the } \\
\text { discontinuation } \\
\text { of } \\
\text { antipsychotics }\end{array}$ & $\begin{array}{l}\text { Community } \\
\text { mental health } \\
\text { centre } \\
\text { outpatient } \\
\text { clinic }\end{array}$ & Schizophrenia & United States \\
\hline
\end{tabular}


Table A1. Cont.

\begin{tabular}{|c|c|c|c|c|c|c|}
\hline Study & Title & Study Design & Concept & Context & Illness & $\begin{array}{l}\text { Country in } \\
\text { Which the } \\
\text { Study } \\
\text { Conducted }\end{array}$ \\
\hline $\begin{array}{l}\text { Vaanholt et al., } \\
2018\end{array}$ & $\begin{array}{c}\text { Perceived } \\
\text { advantages and } \\
\text { disadvantages } \\
\text { of oral } \\
\text { anticoagulants, } \\
\text { and the } \\
\text { trade-offs } \\
\text { patients make } \\
\text { in choosing } \\
\text { anticoagulant } \\
\text { therapy and } \\
\text { adhering to } \\
\text { their drug } \\
\text { regimen }\end{array}$ & Focus group & $\begin{array}{l}\text { Adherence to } \\
\text { oral } \\
\text { anticoagulants }\end{array}$ & $\begin{array}{c}\text { AF patients in } \\
\text { different } \\
\text { European } \\
\text { countries }\end{array}$ & $\begin{array}{c}\text { Cardiovascular } \\
\text { disease }\end{array}$ & Netherland \\
\hline $\begin{array}{l}\text { Van Geffen } \\
\text { et al., } 2011\end{array}$ & $\begin{array}{l}\text { The decision to } \\
\text { continue or } \\
\text { discontinue } \\
\text { treatment: } \\
\text { Experiences } \\
\text { and beliefs of } \\
\text { users of } \\
\text { selective } \\
\text { serotonin- } \\
\text { reuptake } \\
\text { inhibitors in the } \\
\text { initial } \\
\text { months-A } \\
\text { qualitative } \\
\text { study }\end{array}$ & $\begin{array}{l}\text { Individual } \\
\text { interviews }\end{array}$ & $\begin{array}{c}\text { To identify } \\
\text { what reasons } \\
\text { lead to } \\
\text { discontinuation } \\
\text { or continuation } \\
\text { of treatment }\end{array}$ & $\begin{array}{l}\text { Depression } \\
\text { patients in } \\
\text { community } \\
\text { pharmacies } 3 \\
\text { months after } \\
\text { starting SSRI } \\
\text { treatment }\end{array}$ & Depression & Netherland \\
\hline $\begin{array}{c}\text { Van Tam et al., } \\
2011\end{array}$ & $\begin{array}{l}\text { "It is not that I } \\
\text { forget, it's just } \\
\text { that I don't } \\
\text { want other } \\
\text { people to } \\
\text { know": barriers } \\
\text { to and } \\
\text { strategies for } \\
\text { adherence to } \\
\text { antiretroviral } \\
\text { therapy among } \\
\text { HIV patients in } \\
\text { Northern } \\
\text { Vietnam }\end{array}$ & Focus group & $\begin{array}{l}\text { Little is known } \\
\text { about factors } \\
\text { influencing } \\
\text { ART adherence } \\
\text { among people } \\
\text { living with HIV }\end{array}$ & $\begin{array}{l}\text { HIV patients in } \\
\text { Vietnam }\end{array}$ & HIV / AIDS & Vietnam \\
\hline $\begin{array}{l}\text { VanLoggerenberg } \\
\text { et al., } 2015\end{array}$ & $\begin{array}{l}\text { A Qualitative } \\
\text { Study of Patient } \\
\text { Motivation to } \\
\text { Adhere to } \\
\text { Combination } \\
\text { Antiretroviral } \\
\text { Therapy in } \\
\text { South Africa }\end{array}$ & $\begin{array}{c}\text { Other: } \\
\text { Individual } \\
\text { interviews and } \\
\text { focus groups }\end{array}$ & $\begin{array}{c}\text { Adherence to } \\
\text { ART }\end{array}$ & $\begin{array}{l}\text { Patients } \\
\text { receiving ART } \\
\text { medication at } \\
\text { the clinic }\end{array}$ & HIV/AIDS & South Africa \\
\hline
\end{tabular}


Table A1. Cont.

\begin{tabular}{|c|c|c|c|c|c|c|}
\hline Study & Title & Study Design & Concept & Context & Illness & $\begin{array}{l}\text { Country in } \\
\text { Which the } \\
\text { Study } \\
\text { Conducted }\end{array}$ \\
\hline $\begin{array}{l}\text { Verbrugghe } \\
\text { et al., } 2016\end{array}$ & $\begin{array}{c}\text { Factors } \\
\text { influencing } \\
\text { adherence in } \\
\text { cancer patients } \\
\text { taking oral } \\
\text { tyrosine kinase } \\
\text { inhibitors }\end{array}$ & $\begin{array}{l}\text { Individual } \\
\text { interviews }\end{array}$ & $\begin{array}{l}\text { Non-adherence } \\
\text { in cancer } \\
\text { patients taking } \\
\text { oral anticancer } \\
\text { drugs is } \\
\text { common }\end{array}$ & $\begin{array}{l}\text { Five hospitals } \\
\text { in Belgium }\end{array}$ & Cancer & Belgium \\
\hline $\begin{array}{c}\text { Vipey et al., } \\
2021\end{array}$ & $\begin{array}{l}\text { A qualitative } \\
\text { study of } \\
\text { barriers and } \\
\text { facilitators to } \\
\text { adherence to } \\
\text { secondary } \\
\text { prevention } \\
\text { medications } \\
\text { among French } \\
\text { patients } \\
\text { suffering from } \\
\text { stroke and } \\
\text { transient } \\
\text { ischemic attack }\end{array}$ & $\begin{array}{l}\text { Individual } \\
\text { interviews }\end{array}$ & $\begin{array}{l}\text { TIA patients do } \\
\text { not adhere to } \\
\text { their secondary } \\
\text { prevention } \\
\text { medicines }\end{array}$ & $\begin{array}{c}\text { Cohort of TIA } \\
\text { patients in } \\
\text { France }\end{array}$ & $\begin{array}{c}\text { Cardiovascular } \\
\text { disease }\end{array}$ & France \\
\hline $\begin{array}{l}\text { Widayanti et al., } \\
2020\end{array}$ & $\begin{array}{c}\text { Medicine } \\
\text { taking } \\
\text { behaviours of } \\
\text { people with } \\
\text { type } 2 \text { diabetes } \\
\text { in Indonesia: a } \\
\text { qualitative } \\
\text { study }\end{array}$ & Focus group & $\begin{array}{c}\text { Rural and } \\
\text { urban } \\
\text { communities }\end{array}$ & $\begin{array}{l}\text { People's } \\
\text { medicine- } \\
\text { taking } \\
\text { behaviours }\end{array}$ & DM & Indonesia \\
\hline Wu et al., 2015 & $\begin{array}{c}\text { Lack of } \\
\text { congruence } \\
\text { between } \\
\text { patients' and } \\
\text { health } \\
\text { professionals' } \\
\text { perspectives of } \\
\text { adherence to } \\
\text { imatinib } \\
\text { therapy in } \\
\text { treatment of } \\
\text { chronic } \\
\text { myeloid } \\
\text { leukaemia: A } \\
\text { qualitative } \\
\text { study }\end{array}$ & $\begin{array}{l}\text { Individual } \\
\text { interviews }\end{array}$ & $\begin{array}{l}\text { Consistent use } \\
\text { of imatinib is } \\
\text { critical for } \\
\text { treatment } \\
\text { success in } \\
\text { chronic } \\
\text { myeloid } \\
\text { leukaemia }\end{array}$ & $\begin{array}{c}\text { Patients in } \\
\text { specialised } \\
\text { cancer centres } \\
\text { (health } \\
\text { professionals } \\
\text { too) }\end{array}$ & $\begin{array}{l}\text { Chronic } \\
\text { myeloid } \\
\text { leukaemia }\end{array}$ & Australia \\
\hline $\begin{array}{c}\text { Ågärd et al., } \\
2016\end{array}$ & $\begin{array}{l}\text { Diabetes in the } \\
\text { shadow of daily } \\
\text { life: factors that } \\
\text { make diabetes a } \\
\text { marginal } \\
\text { problem }\end{array}$ & $\begin{array}{l}\text { Individual } \\
\text { interviews }\end{array}$ & $\begin{array}{c}\text { Diabetic } \\
\text { patients' } \\
\text { challenges in } \\
\text { following } \\
\text { treatment rec- } \\
\text { ommendations }\end{array}$ & $\begin{array}{l}\text { Medical } \\
\text { outpatient } \\
\text { clinic in } \\
\text { Sweden }\end{array}$ & DM2 & Sweden \\
\hline
\end{tabular}




\section{Appendix C}

Table A2. Summary of systematic reviews.

\begin{tabular}{|c|c|c|c|c|c|c|c|c|}
\hline Study & Title & $\begin{array}{c}\text { Country } \\
\text { in Which } \\
\text { the Study } \\
\text { Con- } \\
\text { ducted }\end{array}$ & $\begin{array}{l}\text { Aim of } \\
\text { Study }\end{array}$ & $\begin{array}{l}\text { Study } \\
\text { Design }\end{array}$ & $\begin{array}{c}\text { Data } \\
\text { Sources }\end{array}$ & $\begin{array}{l}\text { Results According to the } \\
\text { Research Articles }\end{array}$ & $\begin{array}{l}\text { Population } \\
\text { Description }\end{array}$ & $\begin{array}{c}\text { Total } \\
\text { Number of } \\
\text { Participants }\end{array}$ \\
\hline $\begin{array}{l}\text { Al Hamid } \\
\text { et al., } 2014\end{array}$ & $\begin{array}{l}\text { A systematic } \\
\text { review of } \\
\text { qualitative } \\
\text { research on } \\
\text { the } \\
\text { contributory } \\
\text { factors } \\
\text { leading to } \\
\text { medicine- } \\
\text { related } \\
\text { problems } \\
\text { from the } \\
\text { perspectives } \\
\text { of adult } \\
\text { patients with } \\
\text { cardiovascu- } \\
\text { lar diseases } \\
\text { and diabetes } \\
\text { mellitus }\end{array}$ & $\begin{array}{l}12 \\
\text { countries: } \\
\text { Australia, } \\
\text { Brazil, } \\
\text { Cameroon, } \\
\text { Canada, } \\
\text { Croatia, } \\
\text { Ireland, } \\
\text { Malaysia, } \\
\text { South } \\
\text { Africa, } \\
\text { Spain, } \\
\text { Taiwan, } \\
\text { the UK } \\
\text { and the } \\
\text { USA }\end{array}$ & $\begin{array}{l}\text { To explore } \\
\text { and } \\
\text { evaluate } \\
\text { contribu- } \\
\text { tory } \\
\text { factors } \\
\text { leading to } \\
\text { MRPs } \\
\text { among } \\
\text { adult } \\
\text { patients } \\
\text { with CVDs } \\
\text { and/or } \\
\text { DM from } \\
\text { their per- } \\
\text { spectives. }\end{array}$ & $\begin{array}{l}\text { Systematic } \\
\text { review }\end{array}$ & $\begin{array}{l}\text { Pubmed, } \\
\text { EMBASE, } \\
\text { ISIWeb of } \\
\text { Knowl- } \\
\text { edge, } \\
\text { PsycInfo, } \\
\text { Interna- } \\
\text { tional } \\
\text { Pharma- } \\
\text { ceutical } \\
\text { Abstract } \\
\text { and } \\
\text { PsycExtra }\end{array}$ & $\begin{array}{l}\text { Patient-related factors } \\
\text { including socioeconomic } \\
\text { factors (beliefs, feeling } \\
\text { victimised, history of the } \\
\text { condition, lack of finance, } \\
\text { lack of motivation, and } \\
\text { low self-esteem) and } \\
\text { lifestyle factors (diet, lack } \\
\text { of exercise/time to see } \\
\text { the doctor, obesity, } \\
\text { smoking, and stress), } \\
\text { medicine-related factors } \\
\text { (belief in natural } \\
\text { remedies, fear of } \\
\text { medicine, lack of belief in } \\
\text { medicines, lack of } \\
\text { knowledge, non- } \\
\text { adherence, and } \\
\text { polypharmacy) and } \\
\text { condition-related factors } \\
\text { (lack of knowl- } \\
\text { edge/understanding, } \\
\text { fear of condition and its } \\
\text { complications, and lack } \\
\text { of control). }\end{array}$ & $\begin{array}{c}\text { Adult } \\
\text { patients with } \\
\text { cardiovascu- } \\
\text { lar disease } \\
\text { and/or } \\
\text { diabetes }\end{array}$ & $\begin{array}{c}836 \\
\text { (21 studies) }\end{array}$ \\
\hline $\begin{array}{c}\text { Barasa } \\
\text { Masaba } \\
\text { et al., } 2020\end{array}$ & $\begin{array}{l}\text { Determinants } \\
\text { of Non- } \\
\text { Adherence to } \\
\text { Treatment } \\
\text { Among } \\
\text { Patients with } \\
\text { Type } 2 \\
\text { Diabetes in } \\
\text { Kenya: A } \\
\text { Systematic } \\
\text { Review. } \\
\text { [Review] }\end{array}$ & Kenya & $\begin{array}{l}\text { What are } \\
\text { the deter- } \\
\text { minants } \\
\text { that } \\
\text { contribute } \\
\text { to non- } \\
\text { adherence } \\
\text { to } \\
\text { treatment } \\
\text { among } \\
\text { patients } \\
\text { with } \\
\text { T2DM in } \\
\text { Kenya }\end{array}$ & $\begin{array}{l}\text { Systematic } \\
\text { review }\end{array}$ & $\begin{array}{l}\text { Scopus, } \\
\text { Web of } \\
\text { Science, } \\
\text { Science } \\
\text { Direct, } \\
\text { Cochrane } \\
\text { Library, } \\
\text { PUBMED, } \\
\text { OVID and } \\
\text { Google } \\
\text { Scholar. }\end{array}$ & $\begin{array}{l}\text { (1) Cost-income, } \\
\text { insurance, distance, bills } \\
\text { of drugs and food; (2) } \\
\text { Patient characteristics- } \\
\text { perception of (efficacy, } \\
\text { severity, effects of } \\
\text { non-adherence), } \\
\text { knowledge, co-morbidity, } \\
\text { family support, } \\
\text { self-unfounded beliefs; } \\
\text { and (3) Health } \\
\text { system-health } \\
\text { education, multiple } \\
\text { drugs, evaluations and } \\
\text { support, guidelines, and } \\
\text { poor perception of } \\
\text { system. }\end{array}$ & $\begin{array}{c}\text { Adult } \\
\text { patients with } \\
\text { type } 2 \\
\text { diabetes }\end{array}$ & 15 studies \\
\hline
\end{tabular}


Table A2. Cont.

\begin{tabular}{|c|c|c|c|c|c|c|c|c|}
\hline Study & Title & $\begin{array}{c}\text { Country } \\
\text { in Which } \\
\text { the Study } \\
\text { Con- } \\
\text { ducted }\end{array}$ & $\begin{array}{l}\text { Aim of } \\
\text { Study }\end{array}$ & $\begin{array}{l}\text { Study } \\
\text { Design }\end{array}$ & $\begin{array}{l}\text { Data } \\
\text { Sources }\end{array}$ & $\begin{array}{l}\text { Results According to the } \\
\text { Research Articles }\end{array}$ & $\begin{array}{l}\text { Population } \\
\text { Description }\end{array}$ & $\begin{array}{c}\text { Total } \\
\text { Number of } \\
\text { Participants }\end{array}$ \\
\hline $\begin{array}{l}\text { Clancy } \\
\text { et al., } 2020\end{array}$ & $\begin{array}{l}\text { Breast cancer } \\
\text { patients' } \\
\text { experiences } \\
\text { of adherence } \\
\text { and } \\
\text { persistence } \\
\text { to oral } \\
\text { endocrine } \\
\text { therapy: A } \\
\text { qualitative } \\
\text { evidence } \\
\text { synthesis }\end{array}$ & Ireland & $\begin{array}{l}\text { To } \\
\text { synthesise } \\
\text { breast } \\
\text { cancer } \\
\text { patients' } \\
\text { experi- } \\
\text { ences of } \\
\text { adherence } \\
\text { and persis- } \\
\text { tence to } \\
\text { oral } \\
\text { endocrine } \\
\text { therapy. }\end{array}$ & $\begin{array}{l}\text { Systematic } \\
\text { review }\end{array}$ & $\begin{array}{c}\text { Embase, } \\
\text { Cinahl, } \\
\text { Pubmed, } \\
\text { Psychinfo, } \\
\text { Proquest, } \\
\text { Lenus, } \\
\text { Scopus, } \\
\text { Web of } \\
\text { Science, } \\
\text { Rian.ie, } \\
\text { EThOS } \\
\text { e-theses } \\
\text { online, } \\
\text { DART } \\
\text { Europe. } \\
\text { No year } \\
\text { limit } \\
\text { was set }\end{array}$ & $\begin{array}{l}\text { Three analytic themes } \\
\text { were identified (We do } \\
\text { not have an option; the } \\
\text { side effects are worse } \\
\text { than the disease; help us } \\
\text { with information and } \\
\text { support). Non-adherence } \\
\text { and non-persistence were } \\
\text { associated with } \\
\text { debilitating side effects, } \\
\text { inadequate information, } \\
\text { and lack } \\
\text { of support. }\end{array}$ & $\begin{array}{l}\text { Breast cancer } \\
\text { patients }\end{array}$ & 42 studies \\
\hline $\begin{array}{l}\text { Ju et al., } \\
2018\end{array}$ & $\begin{array}{l}\text { Patient } \\
\text { beliefs and } \\
\text { attitudes to } \\
\text { taking } \\
\text { statins: } \\
\text { Systematic } \\
\text { review of } \\
\text { qualitative } \\
\text { studies }\end{array}$ & Australia & $\begin{array}{c}\text { To describe } \\
\text { patients' } \\
\text { perspec- } \\
\text { tives, } \\
\text { experience, } \\
\text { and } \\
\text { attitudes } \\
\text { towards } \\
\text { statins }\end{array}$ & $\begin{array}{l}\text { Systematic } \\
\text { review }\end{array}$ & $\begin{array}{l}\text { PsycINFO, } \\
\text { CINAHL, } \\
\text { Embase, } \\
\text { MEDLINE, } \\
\text { and PhD } \\
\text { disserta- } \\
\text { tions from } \\
\text { inception } \\
\text { to } 6 \\
\text { October } \\
2016\end{array}$ & $\begin{array}{l}\text { Confidence in prevention } \\
\text { (trust in efficacy, } \\
\text { minimising long-term } \\
\text { catastrophic CVD, taking } \\
\text { control, easing anxiety } \\
\text { about high cholesterol); } \\
\text { routinising into daily life; } \\
\text { questioning utility } \\
\text { (imperceptible benefits, } \\
\text { uncertainties about } \\
\text { pharmacological } \\
\text { mechanisms); medical } \\
\text { distrust (scepticism about } \\
\text { overprescribing, pressure } \\
\text { to start therapy); } \\
\text { threatening health } \\
\text { (competing priorities and } \\
\text { risks, debilitating side } \\
\text { effects, toxicity to body); } \\
\text { signifying sickness (fear } \\
\text { of perpetual dependence, } \\
\text { losing the battle); and } \\
\text { financial strain. }\end{array}$ & $\begin{array}{l}\text { cardiovascular } \\
\text { patients } \\
\text { using statins } \\
\text { in different } \\
\text { countries }\end{array}$ & $\begin{array}{c}888 \\
\text { (32 studies) }\end{array}$ \\
\hline
\end{tabular}


Table A2. Cont.

\begin{tabular}{|c|c|c|c|c|c|c|c|c|}
\hline Study & Title & $\begin{array}{c}\text { Country } \\
\text { in Which } \\
\text { the Study } \\
\text { Con- } \\
\text { ducted }\end{array}$ & $\begin{array}{l}\text { Aim of } \\
\text { Study }\end{array}$ & $\begin{array}{l}\text { Study } \\
\text { Design }\end{array}$ & $\begin{array}{c}\text { Data } \\
\text { Sources }\end{array}$ & $\begin{array}{l}\text { Results According to the } \\
\text { Research Articles }\end{array}$ & $\begin{array}{l}\text { Population } \\
\text { Description }\end{array}$ & $\begin{array}{c}\text { Total } \\
\text { Number of } \\
\text { Participants }\end{array}$ \\
\hline $\begin{array}{l}\text { Kelly et al., } \\
2014\end{array}$ & $\begin{array}{l}\text { Knowledge, } \\
\text { attitudes, } \\
\text { and beliefs of } \\
\text { patients and } \\
\text { carers } \\
\text { regarding } \\
\text { medication } \\
\text { adherence: a } \\
\text { review of } \\
\text { qualitative } \\
\text { literature }\end{array}$ & Ireland & $\begin{array}{l}\text { Knowledge, } \\
\text { attitudes, } \\
\text { and beliefs } \\
\text { of patients } \\
\text { and carers } \\
\text { regarding } \\
\text { medica- } \\
\text { tion } \\
\text { adherence }\end{array}$ & $\begin{array}{l}\text { Systematic } \\
\text { review }\end{array}$ & $\begin{array}{c}\text { CINAHL, } \\
\text { Embase, } \\
\text { PubMed, } \\
\text { and Web } \\
\text { of Knowl- } \\
\text { edge from } \\
\text { inception } \\
\text { to } \\
\text { November } \\
2013 .\end{array}$ & $\begin{array}{l}\text { Eight themes were } \\
\text { identified: (1) beliefs and } \\
\text { experiences of medicines, } \\
\text { (2) family support and } \\
\text { culture, (3) role of and } \\
\text { relationship with } \\
\text { healthcare practitioners, } \\
\text { (4) factors related to the } \\
\text { disease, (5) } \\
\text { self-regulation, (6) } \\
\text { communication, (7) cost, } \\
\text { and (8) access. }\end{array}$ & $\begin{array}{l}\text { Users of } \\
\text { medicines } \\
\quad \text { (not } \\
\text { mentioned, } \\
\text { patients in } \\
\text { general) }\end{array}$ & 34 studies \\
\hline $\begin{array}{l}\text { Kelly et al., } \\
2018\end{array}$ & $\begin{array}{c}\text { Patients' } \\
\text { Attitudes } \\
\text { and } \\
\text { Experiences } \\
\text { of Disease- } \\
\text { Modifying } \\
\text { An- } \\
\text { tirheumatic } \\
\text { Drugs in } \\
\text { Rheumatoid } \\
\text { Arthritis and } \\
\text { Spondy- } \\
\text { larthritis: A } \\
\text { Qualitative } \\
\text { Synthesis }\end{array}$ & Australia & $\begin{array}{l}\text { To describe } \\
\text { patients' } \\
\text { attitudes } \\
\text { and experi- } \\
\text { ences of } \\
\text { DMARDS } \\
\text { in RA and } \\
\text { spondy- } \\
\text { larthritis }\end{array}$ & $\begin{array}{l}\text { Systematic } \\
\text { review }\end{array}$ & $\begin{array}{l}\text { MEDLINE, } \\
\text { Embase, } \\
\text { PsycINFO, } \\
\text { and } \\
\text { CINAHL } \\
\text { were } \\
\text { searched } \\
\text { to January } \\
2016\end{array}$ & $\begin{array}{l}\text { Six themes were } \\
\text { identifyed with } \\
\text { subthemes: intensifying } \\
\text { disease identity (severity } \\
\text { of sudden } \\
\text { pharmacotherapy, } \\
\text { signifying deteriorating } \\
\text { health, daunting lifelong } \\
\text { therapy), distressing } \\
\text { uncertainties and } \\
\text { consequences (poisoning } \\
\text { the body, doubting } \\
\text { efficacy, conflicting and } \\
\text { confusing advice, } \\
\text { prognostic uncertainty } \\
\text { with changing treatment } \\
\text { regimens), powerful } \\
\text { social influences (swayed } \\
\text { by others' experiences, } \\
\text { partnering with } \\
\text { physicians, maintaining } \\
\text { roles, confidence in } \\
\text { comprehensive and } \\
\text { ongoing care, valuing } \\
\text { peer support), privilege } \\
\text { and right of access to } \\
\text { biologic agents } \\
\text { (expensive medications } \\
\text { must be better, right to } \\
\text { receive a biologic agent, } \\
\text { fearing dispossession), } \\
\text { maintaining control } \\
\text { (complete ownership of } \\
\text { decision, taking extreme } \\
\text { risks, minimizing life- } \\
\text { style intrusion), and } \\
\text { negotiating treatment } \\
\text { expectations (miraculous } \\
\text { recovery, mediocre } \\
\text { benefit, reaching the end } \\
\text { of the line). }\end{array}$ & $\begin{array}{l}\text { Adults (ages } \\
\geq 18 \text { years) } \\
\text { with } \\
\text { rheumatoid } \\
\text { arthritis or } \\
\text { spondy- } \\
\text { larthritis }\end{array}$ & $\begin{array}{c}1383 \\
\text { (56 studies) }\end{array}$ \\
\hline
\end{tabular}


Table A2. Cont.

\begin{tabular}{|c|c|c|c|c|c|c|c|c|}
\hline Study & Title & $\begin{array}{l}\text { Country } \\
\text { in Which } \\
\text { the Study } \\
\text { Con- } \\
\text { ducted }\end{array}$ & $\begin{array}{l}\text { Aim of } \\
\text { Study }\end{array}$ & $\begin{array}{l}\text { Study } \\
\text { Design }\end{array}$ & $\begin{array}{l}\text { Data } \\
\text { Sources }\end{array}$ & $\begin{array}{l}\text { Results According to the } \\
\text { Research Articles }\end{array}$ & $\begin{array}{l}\text { Population } \\
\text { Description }\end{array}$ & $\begin{array}{c}\text { Total } \\
\text { Number of } \\
\text { Participants }\end{array}$ \\
\hline $\begin{array}{l}\text { Maffoni } \\
\text { et al., } 2020\end{array}$ & $\begin{array}{l}\text { Medication } \\
\text { adherence in } \\
\text { the older } \\
\text { adults with } \\
\text { chronic mul- } \\
\text { timorbidity: } \\
\text { a systematic } \\
\text { review of } \\
\text { qualitative } \\
\text { studies on } \\
\text { patient's } \\
\text { experience }\end{array}$ & $\begin{array}{l}\text { Italy, } \\
\text { Portugal, } \\
\text { Poland }\end{array}$ & $\begin{array}{c}\text { To } \\
\text { investigate } \\
\text { potential } \\
\text { factors } \\
\text { associated } \\
\text { with medi- } \\
\text { cation } \\
\text { adherence } \\
\text { in the } \\
\text { older and } \\
\text { chronic } \\
\text { population } \\
\text { through a } \\
\text { PRISMA } \\
\text { systematic } \\
\text { review of } \\
\text { qualitative } \\
\text { studies on } \\
\text { patients' } \\
\text { experi- } \\
\text { ence. }\end{array}$ & $\begin{array}{l}\text { Systematic } \\
\text { review }\end{array}$ & $\begin{array}{c}\text { Scopus } \\
\text { and } \\
\text { Pubmed } \\
\text { from } 2000 \\
\text { to October } \\
2017\end{array}$ & $\begin{array}{l}\text { According to the ABC } \\
\text { Taxonomy, Persistence } \\
\text { and Implementation were } \\
\text { the most often considered } \\
\text { phases. Considering the } \\
\text { Three Factor model, the } \\
\text { most often reported } \\
\text { themes were Information } \\
\text { and Strategies upon } \\
\text { being adherent. The } \\
\text { patient's decisional } \\
\text { flowchart describing } \\
\text { barriers and facilitators } \\
\text { (personal, social and } \\
\text { environmental) to } \\
\text { adherence was proposed. }\end{array}$ & $\begin{array}{l}\text { Older adults } \\
\text { aged } 65 \text { or } \\
\text { more }\end{array}$ & 39 studies \\
\hline $\begin{array}{l}\text { Marshall } \\
\text { et al., } 2012\end{array}$ & $\begin{array}{c}\text { Lay } \\
\text { perspectives } \\
\text { on hyperten- } \\
\text { sion and } \\
\text { drug } \\
\text { adherence: } \\
\text { systematic } \\
\text { review of } \\
\text { qualitative } \\
\text { research }\end{array}$ & UK & $\begin{array}{c}\text { To } \\
\text { examine } \\
\text { lay under- } \\
\text { standing } \\
\text { of hyper- } \\
\text { tension } \\
\text { and per- } \\
\text { spectives } \\
\text { on drug } \\
\text { taking }\end{array}$ & $\begin{array}{l}\text { Systematic } \\
\text { review }\end{array}$ & $\begin{array}{c}\text { Medline, } \\
\text { Embase, } \\
\text { the British } \\
\text { Nursing } \\
\text { Index, } \\
\text { Social } \\
\text { Policy and } \\
\text { Practice, } \\
\text { and } \\
\text { PsycInfo } \\
\text { from } \\
\text { inception } \\
\text { to October } \\
2011\end{array}$ & $\begin{array}{l}\text { A large proportion of } \\
\text { participants thought } \\
\text { hypertension was } \\
\text { principally caused by } \\
\text { stress and produced } \\
\text { symptoms, particularly } \\
\text { headache, dizziness, and } \\
\text { sweating. Participants } \\
\text { widely intentionally } \\
\text { reduced or stopped } \\
\text { treatment without } \\
\text { consulting their doctor, } \\
\text { commonly perceived that } \\
\text { their blood pressure } \\
\text { improved when } \\
\text { symptoms abated or } \\
\text { when they were not } \\
\text { stressed, and that } \\
\text { treatment was not } \\
\text { needed at these times. } \\
\text { Participants disliked } \\
\text { treatment and its side } \\
\text { effects and feared } \\
\text { addiction. Participants } \\
\text { reported various external } \\
\text { factors that prevented } \\
\text { adherence: being unable } \\
\text { to find time to take the } \\
\text { drugs or to see the doctor; } \\
\text { having insufficient } \\
\text { money to pay for } \\
\text { treatment; the cost of } \\
\text { appointments and } \\
\text { healthy food; a lack of } \\
\text { health insurance; and } \\
\text { forgetfulness. }\end{array}$ & $\begin{array}{l}\text { A global } \\
\text { population } \\
\text { with cardio- } \\
\text { vascular } \\
\text { disease }\end{array}$ & $\begin{array}{c}53 \\
\text { qualitative } \\
\text { studies }\end{array}$ \\
\hline
\end{tabular}


Table A2. Cont.

\begin{tabular}{|c|c|c|c|c|c|c|c|c|}
\hline Study & Title & $\begin{array}{c}\text { Country } \\
\text { in Which } \\
\text { the Study } \\
\text { Con- } \\
\text { ducted }\end{array}$ & $\begin{array}{l}\text { Aim of } \\
\text { Study }\end{array}$ & $\begin{array}{l}\text { Study } \\
\text { Design }\end{array}$ & $\begin{array}{c}\text { Data } \\
\text { Sources }\end{array}$ & $\begin{array}{l}\text { Results According to the } \\
\text { Research Articles }\end{array}$ & $\begin{array}{l}\text { Population } \\
\text { Description }\end{array}$ & $\begin{array}{c}\text { Total } \\
\text { Number of } \\
\text { Participants }\end{array}$ \\
\hline $\begin{array}{l}\text { McSharry } \\
\text { et al., } 2016\end{array}$ & $\begin{array}{l}\text { Systematic } \\
\text { Review or } \\
\text { Meta- } \\
\text { analysis } \\
\text { Perceptions } \\
\text { and } \\
\text { experiences } \\
\text { of taking oral } \\
\text { medications } \\
\text { for the } \\
\text { treatment of } \\
\text { Type } 2 \\
\text { diabetes } \\
\text { mellitus: a } \\
\text { systematic } \\
\text { review and } \\
\text { meta- } \\
\text { synthesis of } \\
\text { qualitative } \\
\text { studies }\end{array}$ & UK & $\begin{array}{c}\text { To explore } \\
\text { patients' } \\
\text { percep- } \\
\text { tions and } \\
\text { experi- } \\
\text { ences of } \\
\text { taking oral } \\
\text { medica- } \\
\text { tions for } \\
\text { the } \\
\text { pharmaco- } \\
\text { logical } \\
\text { manage- } \\
\text { ment of } \\
\text { Type } 2 \\
\text { diabetes by } \\
\text { carrying } \\
\text { out a } \\
\text { systematic } \\
\text { review and } \\
\text { qualitative } \\
\text { meta- } \\
\text { synthesis } \\
\text { of } \\
\text { published } \\
\text { qualitative } \\
\text { studies }\end{array}$ & $\begin{array}{l}\text { Systematic } \\
\text { review }\end{array}$ & $\begin{array}{c}\text { Cinahl, } \\
\text { EMBASE, } \\
\text { Medline, } \\
\text { and } \\
\text { PsycINFO } \\
\text { databases } \\
\text { were } \\
\text { searched } \\
\text { in } 2014\end{array}$ & $\begin{array}{l}\text { Medications for diabetes: } \\
\text { a necessary evil, outlines } \\
\text { how patients' negative } \\
\text { perceptions of medication } \\
\text { risks co-exist with a } \\
\text { resounding view that } \\
\text { medications are } \\
\text { beneficial. Passive } \\
\text { patients but active } \\
\text { experimenters highlights } \\
\text { the contrast between } \\
\text { patients' passive } \\
\text { acceptance of medication } \\
\text { prescriptions and the } \\
\text { urge to actively } \\
\text { experiment and adjust } \\
\text { doses to optimize } \\
\text { medication use in daily } \\
\text { life. Finally, Taking oral } \\
\text { medication for Type } 2 \\
\text { diabetes: a unique } \\
\text { context describes features } \\
\text { specific to the Type } 2 \\
\text { diabetes medication } \\
\text { experience, including } \\
\text { lack of symptoms and the } \\
\text { perceived relationship } \\
\text { between medication and } \\
\text { diet, which may influence } \\
\text { adherence. }\end{array}$ & $\begin{array}{l}\text { Diabetes } \\
\text { type } 2 \\
\text { patients } \\
\text { taking oral } \\
\text { medication }\end{array}$ & 8 studies \\
\hline $\begin{array}{l}\text { Nielsen } \\
\text { et al., } 2018\end{array}$ & $\begin{array}{l}\text { Adherence } \\
\text { to } \\
\text { medication } \\
\text { in patients } \\
\text { with chronic } \\
\text { kidney } \\
\text { disease: a } \\
\text { systematic } \\
\text { review of } \\
\text { qualitative } \\
\text { research }\end{array}$ & Denmark & $\begin{array}{c}\text { To } \\
\text { synthesize } \\
\text { findings } \\
\text { from } \\
\text { qualitative } \\
\text { studies of } \\
\text { patients' } \\
\text { experi- } \\
\text { ences of } \\
\text { factors that } \\
\text { facilitate } \\
\text { and hinder } \\
\text { adherence } \\
\text { to medica- } \\
\text { tion. }\end{array}$ & $\begin{array}{l}\text { Systematic } \\
\text { review }\end{array}$ & $\begin{array}{l}\text { MEDLINE, } \\
\text { Embase, } \\
\text { and } \\
\text { CINAHL }\end{array}$ & $\begin{array}{l}\text { Three analytical themes } \\
\text { with the subthemes; }(1) \\
\text { logistics (establishing and } \\
\text { maintaining routines, } \\
\text { and the costs of buying } \\
\text { medication), (2) } \\
\text { benchmarking the need } \\
\text { for medication (absence } \\
\text { of effect from a lay } \\
\text { perspective, lacking } \\
\text { understanding of } \\
\text { medication indications } \\
\text { and effects and being } \\
\text { spurred by emergent } \\
\text { symptoms) and (3) the } \\
\text { quality of the patient- } \\
\text { physician relationship } \\
\text { (eliciting patients' wishes } \\
\text { for involvement in } \\
\text { decisions concerning } \\
\text { medication and lacking } \\
\text { information). }\end{array}$ & $\begin{array}{c}\text { Adult } \\
\text { patients with } \\
\text { chronic } \\
\text { kidney } \\
\text { disease }\end{array}$ & $\begin{array}{c}381 \\
\text { (19 studies) }\end{array}$ \\
\hline
\end{tabular}


Table A2. Cont.

\begin{tabular}{|c|c|c|c|c|c|c|c|c|}
\hline Study & Title & $\begin{array}{c}\text { Country } \\
\text { in Which } \\
\text { the Study } \\
\text { Con- } \\
\text { ducted }\end{array}$ & $\begin{array}{l}\text { Aim of } \\
\text { Study }\end{array}$ & $\begin{array}{l}\text { Study } \\
\text { Design }\end{array}$ & $\begin{array}{c}\text { Data } \\
\text { Sources }\end{array}$ & $\begin{array}{l}\text { Results According to the } \\
\text { Research Articles }\end{array}$ & $\begin{array}{l}\text { Population } \\
\text { Description }\end{array}$ & $\begin{array}{c}\text { Total } \\
\text { Number of } \\
\text { Participants }\end{array}$ \\
\hline $\begin{array}{l}\text { Rashid } \\
\text { et al., } 2014\end{array}$ & $\begin{array}{l}\text { Medication } \\
\text { taking in } \\
\text { coronary } \\
\text { artery } \\
\text { disease: A } \\
\text { systematic } \\
\text { review and } \\
\text { qualitative } \\
\text { synthesis }\end{array}$ & UK & $\begin{array}{l}\text { To under- } \\
\text { stand from } \\
\text { a patient } \\
\text { perspec- } \\
\text { tive the } \\
\text { factors that } \\
\text { promote } \\
\text { medica- } \\
\text { tion } \\
\text { persis- } \\
\text { tence. }\end{array}$ & $\begin{array}{l}\text { Systematic } \\
\text { review }\end{array}$ & $\begin{array}{c}\text { MEDLINE, } \\
\text { Embase, } \\
\text { Psy- } \\
\text { cINFO, } \\
\text { SCOPUS, } \\
\text { CINAHL, } \\
\text { ASSIA, } \\
\text { and SSCI }\end{array}$ & $\begin{array}{l}\text { Some patients hold } \\
\text { fatalistic beliefs about } \\
\text { their disease, whereas } \\
\text { others believe they have } \\
\text { been cured by } \\
\text { interventions; both can } \\
\text { lead to failure to take } \\
\text { medication. Patients who } \\
\text { adapt to being a "heart } \\
\text { patient" are positive } \\
\text { about medication taking. } \\
\text { Some individuals dislike } \\
\text { taking tablets generally } \\
\text { and are wary of } \\
\text { long-term effects. } \\
\text { Relationships with } \\
\text { prescribing clinicians are } \\
\text { of critical importance for } \\
\text { patients, with } \\
\text { inaccessibility and } \\
\text { insensitive terminology } \\
\text { negatively affecting } \\
\text { patients' perceptions } \\
\text { about treatments. }\end{array}$ & $\begin{array}{c}\text { Adult } \\
\text { patients with } \\
\text { cardiovascu- } \\
\text { lar } \\
\text { disease }\end{array}$ & $\begin{array}{c}391 \\
\text { (17 studies) }\end{array}$ \\
\hline $\begin{array}{l}\text { Rathbone } \\
\text { et al., } 2017\end{array}$ & $\begin{array}{l}\text { A systematic } \\
\text { review and } \\
\text { thematic } \\
\text { synthesis of } \\
\text { patients' } \\
\text { experience of } \\
\text { medicines } \\
\text { adherence }\end{array}$ & UK & $\begin{array}{l}\text { To explore } \\
\text { patients' } \\
\text { lived expe- } \\
\text { riences of } \\
\text { medicines } \\
\text { adherence } \\
\text { reported in } \\
\text { the phe- } \\
\text { nomeno- } \\
\text { logical } \\
\text { literature, } \\
\text { through } \\
\text { systematic } \\
\text { review and } \\
\text { thematic } \\
\text { synthesis }\end{array}$ & $\begin{array}{l}\text { Systematic } \\
\text { review }\end{array}$ & $\begin{array}{l}\text { CINAHL, } \\
\text { PsychInfo, } \\
\text { Web of } \\
\text { Science, So- } \\
\text { ciological } \\
\text { Abstracts, } \\
\text { MEDLINE }\end{array}$ & $\begin{array}{l}\text { Descriptive themes } \\
\text { identified included (1) } \\
\text { dislike for medicines, (2) } \\
\text { survival, (3) perceived } \\
\text { need, including (a) } \\
\text { symptoms and } \\
\text { side-effects and (b) cost, } \\
\text { and (4) routine. Analytic } \\
\text { themes identified were (1) } \\
\text { identity and (2) } \\
\text { interaction. }\end{array}$ & $\begin{array}{c}\text { Adult } \\
\text { patients with } \\
\text { cardiovascu- } \\
\text { lar } \\
\text { disease }\end{array}$ & $\begin{array}{c}463 \\
\text { (22 studies) }\end{array}$ \\
\hline $\begin{array}{l}\text { Tong et al., } \\
2011\end{array}$ & $\begin{array}{l}\text { The } \\
\text { perspectives } \\
\text { of kidney } \\
\text { transplant } \\
\text { recipients on } \\
\text { medicine } \\
\text { taking: A } \\
\text { systematic } \\
\text { review of } \\
\text { qualitative } \\
\text { studies }\end{array}$ & Australia & $\begin{array}{c}\text { To } \\
\text { summarise } \\
\text { and } \\
\text { synthesise } \\
\text { published } \\
\text { qualitative } \\
\text { studies on } \\
\text { the experi- } \\
\text { ences, } \\
\text { perspec- } \\
\text { tives, } \\
\text { beliefs and } \\
\text { attitudes } \\
\text { of kidney } \\
\text { transplant } \\
\text { recipients } \\
\text { on } \\
\text { medicine } \\
\text { taking. }\end{array}$ & $\begin{array}{l}\text { Systematic } \\
\text { review }\end{array}$ & $\begin{array}{c}\text { Medline, } \\
\text { PsycINFO, } \\
\text { EMBASE, } \\
\text { Cochrane } \\
\text { Database } \\
\text { from } \\
\text { inception } \\
\text { until Week } \\
3 \text { of } \\
\text { January } \\
2010\end{array}$ & $\begin{array}{l}\text { (1) attitudes towards } \\
\text { medicine taking, its } \\
\text { impact on lifestyle, } \\
\text { self-image, relationships } \\
\text { and outlook on life; (2) } \\
\text { inadvertent forgetfulness, } \\
\text { preoccupation with life } \\
\text { commitments; (3) } \\
\text { medication properties; (4) } \\
\text { structure of healthcare } \\
\text { services, poor access to } \\
\text { pharmacy or affordable } \\
\text { medications and } \\
\text { conflicting medical } \\
\text { appointments; (5) } \\
\text { personal efforts in } \\
\text { managing medications, } \\
\text { organizing and devising } \\
\text { strategies for taking } \\
\text { medicines on time; and } \\
\text { (6) availability of external } \\
\text { social support }\end{array}$ & $\begin{array}{c}\text { Adult } \\
\text { patients with } \\
\text { kidney } \\
\text { transplants }\end{array}$ & $\begin{array}{c}207 \\
\text { (7 studies) }\end{array}$ \\
\hline
\end{tabular}




\section{References}

1. Eduardo, S. Adherence to Long-Term Therapies: Evidence for Action; World Health Organization: Geneva, Switzerland, 2003.

2. Haynes, R.B.; Ackloo, E.; Sahota, N.; McDonald, H.P.; Yao, X. Interventions for enhancing medication adherence. Cochrane Database Syst. Rev. 2008. [CrossRef]

3. Nieuwlaat, R.; Wilczynski, N.; Navarro, T.; Hobson, N.; Jeffery, R.; Keepanasseril, A.; Agoritsas, T.; Mistry, N.; Iorio, A.; Jack, S.; et al. Interventions for enhancing medication adherence. Cochrane Database Syst. Rev. 2014. [CrossRef]

4. Amankwaa, I.; Boateng, D.; Quansah, D.Y.; Akuoko, C.P.; Evans, C. Effectiveness of short message services and voice call interventions for antiretroviral therapy adherence and other outcomes: A systematic review and meta-analysis. PLoS ONE 2018, 13, e0204091. [CrossRef]

5. Rasmussen, J.N.; Chong, A.; Alter, D.A. Relationship between adherence to evidence-based pharmacotherapy and long-term mortality after acute myocardial infarction. JAMA 2007, 297, 177-186. [CrossRef]

6. Roebuck, M.C.; Liberman, J.N.; Gemmill-Toyama, M.; Brennan, T.A. Medication adherence leads to lower health care use and costs despite increased drug spending. Health Aff. 2011, 30, 91-99. [CrossRef] [PubMed]

7. Iuga, A.O.; McGuire, M.J. Adherence and health care costs. Risk Manag. Healthc. Policy 2014, 7, 35-44. [CrossRef] [PubMed]

8. Cutler, R.L.; Fernandez-Llimos, F.; Frommer, M.; Benrimoj, C.; Garcia-Cardenas, V. Economic impact of medication non-adherence by disease groups: A systematic review. BMJ Open 2018, 8, e016982. [CrossRef] [PubMed]

9. Bauler, S.; Jacquin-Courtois, S.; Haesebaert, J.; Luaute, J.; Coudeyre, E.; Feutrier, C.; Allenet, B.; Decullier, E.; Rode, G.; JanolyDumenil, A. Barriers and facilitators for medication adherence in stroke patients: A qualitative study conducted in french neurological rehabilitation units. Eur. Neurol. 2014, 72, 262-270. [CrossRef] [PubMed]

10. Nielsen, T.M.; Juhl, M.F.; Feldt-Rasmussen, B.; Thomsen, T. Adherence to medication in patients with chronic kidney disease: A systematic review of qualitative research. Clin. Kidney J. 2018, 11, 513-527. [CrossRef]

11. Rezaei, M.; Valiee, S.; Tahan, M.; Ebtekar, F.; Gheshlagh, R.G. Barriers of medication adherence in patients with type-2 diabetes: A pilot qualitative study. Diabetes Metab. Syndr. Obes. Targets Ther. 2019, 12, 589-599. [CrossRef]

12. Mohammed, M.A.; Moles, R.J.; Chen, T.F. Medication-related burden and patients' lived experience with medicine: A systematic review and metasynthesis of qualitative studies. BMJ Open 2016, 6. [CrossRef] [PubMed]

13. Herrera, P.A.; Moncada, L.; Defey, D. Understanding Non-Adherence From the Inside: Hypertensive Patients' Motivations for Adhering and Not Adhering. Qual. Health Res. 2017, 27, 1023-1034. [CrossRef]

14. Pagès-Puigdemont, N.; Mangues, M.A.; Masip, M.; Gabriele, G.; Fernández-Maldonado, L.; Blancafort, S.; Tuneu, L. Patients' Perspective of Medication Adherence in Chronic Conditions: A Qualitative Study. Adv. Ther. 2016, 33, 1740-1754. [CrossRef] [PubMed]

15. Fisher, J.D.; Fisher, W.A. Changing AIDS-risk behavior. Psychol Bull. 1992, 111, 455-474. [CrossRef]

16. Yang, C.; Hui, Z.; Zeng, D.; Liu, L.; Lee, D.T.F. Examining and adapting the information-motivation-behavioural skills model of medication adherence among community-dwelling older patients with multimorbidity: Protocol for a cross-sectional study. BMJ Open 2020, 10, e033431. [CrossRef] [PubMed]

17. Carpenter, D.M.; DeVellis, R.F.; Fisher, E.B.; DeVellis, B.M.; Hogan, S.L.; Jordan, J.M. The effect of conflicting medication information and physician support on medication adherence for chronically ill patients. Patient Educ. Couns. 2010, 81, 169-176. [CrossRef] [PubMed]

18. Reason, J. Human error: Models and management. BMJ 2000, 320, 768-770. [CrossRef]

19. Tricco, A.C.; Lillie, E.; Zarin, W.; O’Brien, K.K.; Colquhoun, H.; Levac, D.; Moher, D.; Peters, M.D.J.; Horsley, T.; Weeks, L.; et al. PRISMA Extension for Scoping Reviews (PRISMA-ScR): Checklist and Explanation. Ann. Intern. Med. 2018, 169, 467-473. [CrossRef]

20. Peters, M.D.J.; Marnie, C.; Tricco, A.C.; Pollock, D.; Munn, Z.; Alexander, L.; McInerney, P.; Godfrey, C.M.; Khalil, H. Updated methodological guidance for the conduct of scoping reviews. JBI Evid. Implement. 2021, 19, 3-10. [CrossRef]

21. Al Hamid, A.; Ghaleb, M.; Aljadhey, H.; Aslanpour, Z. A systematic review of qualitative research on the contributory factors leading to medicine-related problems from the perspectives of adult patients with cardiovascular diseases and diabetes mellitus. BMJ Open 2014, 4. [CrossRef]

22. Ju, A.; Hanson, C.S.; Banks, E.; Korda, R.; Craig, J.C.; Usherwood, T.; MacDonald, P.; Tong, A. Patient beliefs and attitudes to taking statins: Systematic review of qualitative studies. Br. J. Gen. Pract. 2018, 68, e408-e419. [CrossRef]

23. Marshall, I.J.; Wolfe, C.D.A.; McKevitt, C. Lay perspectives on hypertension and drug adherence: Systematic review of qualitative research. BMJ 2012, 345. [CrossRef] [PubMed]

24. Rashid, M.A.; Edwards, D.; Walter, F.M.; Mant, J. Medication taking in coronary artery disease: A systematic review and qualitative synthesis. Ann. Fam. Med. 2014, 12, 224-232. [CrossRef]

25. Rathbone, A.P.; Todd, A.; Jamie, K.; Bonam, M.; Banks, L.; Husband, A.K. A systematic review and thematic synthesis of patients experience of medicines adherence. Res. Soc. Adm. Pharm. 2017, 13, 403-439. [CrossRef] [PubMed]

26. Bellack, A.S.; Bowden, C.L.; Bowie, C.R.; Byerly, M.J.; Carpenter, W.T.; Copeland, L.A.; Dassori, A.M.; Davis, J.M.; Depp, C.A.; Diaz, E.; et al. The expert consensus guideline series: Adherence problems in patients with serious and persistent mental illness. J. Clin. Psychiatry 2009, 70, 1-48.

27. Barasa Masaba, B.; Mmusi-Phetoe, R.M. Determinants of Non-Adherence to Treatment Among Patients with Type 2 Diabetes in Kenya: A Systematic Review. J. Multidiscip. Healthc. 2020, 13, 2069-2076. [CrossRef] [PubMed] 
28. Kelly, A.; Tymms, K.; Tunnicliffe, D.J.; Sumpton, D.; Perera, C.; Fallon, K.; Craig, J.C.; Abhayaratna, W.; Tong, A. Patients' Attitudes and Experiences of Disease-Modifying Antirheumatic Drugs in Rheumatoid Arthritis and Spondyloarthritis: A Qualitative Synthesis. Arthritis Care Res. 2018, 70, 525-532. [CrossRef] [PubMed]

29. Clancy, C.; Lynch, J.; OConnor, P.; Dowling, M. Breast cancer patients' experiences of adherence and persistence to oral endocrine therapy: A qualitative evidence synthesis. Eur. J. Oncol. Nurs. 2020, 44, 101706. [CrossRef] [PubMed]

30. Tong, A.; Howell, M.; Wong, G.; Webster, A.C.; Howard, K.; Craig, J.C. The perspectives of kidney transplant recipients on medicine taking: A systematic review of qualitative studies. Nephrol. Dial. Transplant. 2011, 26, 344-354. [CrossRef] [PubMed]

31. Kelly, M.; McCarthy, S.; Sahm, L.J. Knowledge, attitudes and beliefs of patients and carers regarding medication adherence: A review of qualitative literature. Eur. J. Clin. Pharmacol. 2014, 70, 1423-1431. [CrossRef]

32. Maffoni, M.; Traversoni, S.; Costa, E.; Midao, L.; Kardas, P.; Kurczewska-Michalak, M.; Giardini, A. Medication adherence in the older adults with chronic multimorbidity: A systematic review of qualitative studies on patient's experience. Eur. Geriatr. Med. 2020, 11, 369-381. [CrossRef]

33. Holtzman, C.W.; Shea, J.A.; Glanz, K.; Jacobs, L.M.; Gross, R.; Hines, J.; Mounzer, K.; Samuel, R.; Metlay, J.P.; Yehia, B.R. Mapping patient-identified barriers and facilitators to retention in HIV care and antiretroviral therapy adherence to Andersen's Behavioral Model. AIDS Care Psychol. Socio-Med. Asp. AIDS/HIV 2015, 27, 817-828. [CrossRef]

34. Schatz, E.; Seeley, J.; Negin, J.; Weiss, H.A.; Tumwekwase, G.; Kabunga, E.; Nalubega, P.; Mugisha, J. “for us here, we remind ourselves": Strategies and barriers to ART access and adherence among older Ugandans. BMC Public Health 2019, 19. [CrossRef]

35. Bockwoldt, D.; Staffileno, B.A.; Coke, L.; Hamilton, R.; Fogg, L.; Calvin, D.; Quinn, L. Understanding Experiences of Diabetes Medications Among African Americans Living With Type 2 Diabetes. J. Transcult. Nurs. 2017, 28, 363-371. [CrossRef] [PubMed]

36. McDonald, S.; Ferguson, E.; Hagger, M.S.; Foss, A.J.E.; King, A.J. A theory-driven qualitative study exploring issues relating to adherence to topical glaucoma medications. Patient Prefer. Adherence 2019, 13, 819-828. [CrossRef]

37. Pettersen, T.R.; Fridlund, B.; Bendz, B.; Nordrehaug, J.E.; Rotevatn, S.; Schjøtt, J.; Norekvål, T.M.; On behalf of the, C.I. Challenges adhering to a medication regimen following first-time percutaneous coronary intervention: A patient perspective. Int. J. Nurs. Stud. 2018, 88, 16-24. [CrossRef] [PubMed]

38. Becker, N.; Cordeiro, L.S.; Poudel, K.C.; Sibiya, T.E.; Sayer, A.G.; Sibeko, L.N. Individual, household, and community level barriers to ART adherence among women in rural Eswatini. PLoS ONE 2020, 15. [CrossRef] [PubMed]

39. Lambert-Kerzner, A.; Havranek, E.P.; Plomondon, M.E.; Fagan, K.M.; McCreight, M.S.; Fehling, K.B.; Williams, D.J.; Hamilton, A.B.; Albright, K.; Blatchford, P.J.; et al. Perspectives of patients on factors relating to adherence to post-acute coronary syndrome medical regimens. Patient Prefer. Adherence 2015, 9, 1053-1059. [CrossRef] [PubMed]

40. Kucukarslan, S.N.; Lewis, N.J.W.; Shimp, L.A.; Gaither, C.A.; Lane, D.C.; Baumer, A.L. Exploring patient experiences with prescription medicines to identify unmet patient needs: Implications for research and practice. Res. Soc. Adm. Pharm. 2012, 8, 321-332. [CrossRef] [PubMed]

41. Huang, Y.M.; Pecanac, K.E.; Shiyanbola, O.O. “Why Am I Not Taking Medications?” Barriers and Facilitators of Diabetes Medication Adherence Across Different Health Literacy Levels. Qual. Health Res. 2020, 30, 2331-2342. [CrossRef]

42. Garavalia, L.; Garavalia, B.; Spertus, J.A.; Decker, C. Exploring patients' reasons for discontinuance of heart medications. J. Cardiovasc. Nurs. 2009, 24, 371-379. [CrossRef] [PubMed]

43. Garavalia, L.; Ho, P.M.; Garavalia, B.; Foody, J.M.; Kruse, H.; Spertus, J.A.; Decker, C. Clinician-patient discord: Exploring differences in perspectives for discontinuing clopidogrel. Eur. J. Cardiovasc. Nurs. 2011, 10, 50-55. [CrossRef]

44. Oshotse, C.; Zullig, L.L.; Bosworth, H.B.; Tu, P.; Lin, C. Self-efficacy and adherence behaviors in rheumatoid arthritis patients. Prev. Chronic Dis. 2018, 15. [CrossRef] [PubMed]

45. Rahmawati, R.; Bajorek, B. Understanding untreated hypertension from patients' point of view: A qualitative study in rural Yogyakarta province, Indonesia. Chronic Illn. 2018, 14, 228-240. [CrossRef]

46. Meraz, R. Medication Nonadherence or Self-care? Understanding the Medication Decision-Making Process and Experiences of Older Adults With Heart Failure. J. Cardiovasc. Nurs. 2020, 35, 26-34. [CrossRef]

47. Sapkota, S.; Brien, J.A.E.; Aslani, P. Nepalese patients' anti-diabetic medication taking behaviour: An exploratory study. Ethn. Health 2018, 23, 718-736. [CrossRef] [PubMed]

48. Evon, D.M.; Golin, C.E.; Bonner, J.E.; Grodensky, C.; Velloza, J. Adherence during antiviral treatment regimens for chronic Hepatitis C. J. Clin. Gastroenterol. 2015, 49, e41-e50. [CrossRef] [PubMed]

49. Axelsson, J.M.; Hallager, S.; Barfod, T.S. Antiretroviral therapy adherence strategies used by patients of a large HIV clinic in Lesotho. J. Health Popul. Nutr. 2015, 33. [CrossRef]

50. Al-Qazaz, H.K.; Hassali, M.A.; Shafie, A.A.; Syed Sulaiman, S.A.; Sundram, S. Perception and knowledge of patients with type 2 diabetes in Malaysia about their disease and medication: A qualitative study. Res. Soc. Adm. Pharm. 2011, 7, 180-191. [CrossRef]

51. Tranulis, C.; Goff, D.; Henderson, D.C.; Freudenreich, O. Becoming adherent to antipsychotics: A qualitative study of treatmentexperienced schizophrenia patients. Psychiatr. Serv. 2011, 62, 888-892. [CrossRef]

52. Ågärd, A.; Ranjbar, V.; Strang, S. Diabetes in the shadow of daily life: Factors that make diabetes a marginal problem. Pract. Diabetes 2016, 33, 49-53. [CrossRef]

53. Shaw, Y.; Metes, I.D.; Michaud, K.; Donohue, J.M.; Roberts, M.S.; Levesque, M.C.; Chang, J.C. Rheumatoid Arthritis Patients' Motivations for Accepting or Resisting Disease-Modifying Antirheumatic Drug Treatment Regimens. Arthritis Care Res. 2018, 70, 533-541. [CrossRef] 
54. Stryker, J.E.; Beck, A.D.; Primo, S.A.; Echt, K.V.; Bundy, L.; Pretorius, G.C.; Glanz, K. An exploratory study of factors influencing glaucoma treatment adherence. J. Glaucoma 2010, 19, 66-72. [CrossRef]

55. Ho, S.C.; Jacob, S.A.; Tangiisuran, B. Barriers and facilitators of adherence to antidepressants among outpatients with major depressive disorder: A qualitative study. PLoS ONE 2017, 12. [CrossRef] [PubMed]

56. Rowell-Cunsolo, T.L.; Hu, G. Barriers to optimal antiretroviral therapy adherence among HIV-infected formerly incarcerated individuals in New York City. PLoS ONE 2020, 15. [CrossRef] [PubMed]

57. Koh, J.J.K.; Cheng, R.X.; Yap, Y.; Haldane, V.; Tan, Y.G.; Teo, K.W.Q.; Srivastava, A.; Ong, P.S.; Perel, P.; Legido-Quigley, H. Access and adherence to medications for the primary and secondary prevention of atherosclerotic cardiovascular disease in singapore: A qualitative study. Patient Prefer. Adherence 2018, 12, 2481-2498. [CrossRef] [PubMed]

58. Jarab, A.S.; Mukattash, T.L.; Al-Azayzih, A.; Khdour, M. A focus group study of patient's perspective and experiences of type 2 diabetes and its management in Jordan. Saudi Pharm. J. 2018, 26, 301-305. [CrossRef]

59. Gassmann, C.; Kolbe, N.; Brenner, A. Experiences and coping strategies of oncology patients undergoing oral chemotherapy: First steps of a grounded theory study. Eur. J. Oncol. Nurs. 2016, 23, 106-114. [CrossRef] [PubMed]

60. Habte, B.M.; Kebede, T.; Fenta, T.G.; Boon, H. Barriers and facilitators to adherence to anti-diabetic medications: Ethiopian patients' perspectives. Afr. J. Prim. Health Care Fam. Med. 2017, 9. [CrossRef]

61. Harrold, L.R.; Mazor, K.M.; Velten, S.; Ockene, I.S.; Yood, R.A. Patients and providers view gout differently: A qualitative study. Chronic Illn. 2010, 6, 263-271. [CrossRef]

62. Hogan, A.; Bonney, M.A.; Brien, J.A.; Karamy, R.; Aslani, P. Factors affecting nebulised medicine adherence in adult patients with cystic fibrosis: A qualitative study. Int. J. Clin. Pharm. 2014, 37, 86-93. [CrossRef]

63. Vaanholt, M.C.W.; Weernink, M.G.M.; von Birgelen, C.; Groothuis-Oudshoorn, C.G.M.; MJ, I.J.; van Til, J.A. Perceived advantages and disadvantages of oral anticoagulants, and the trade-offs patients make in choosing anticoagulant therapy and adhering to their drug regimen. Patient Educ. Couns. 2018, 101, 1982-1989. [CrossRef] [PubMed]

64. King-Shier, K.M.; Singh, S.; Khan, N.A.; LeBlanc, P.; Lowe, J.C.; Mather, C.M.; Chong, E.; Quan, H. Ethno-Cultural Considerations in Cardiac Patients' Medication Adherence. Clin. Nurs. Res. 2017, 26, 576-591. [CrossRef]

65. Van Loggerenberg, F.; Gray, D.; Gengiah, S.; Kunene, P.; Gengiah, T.N.; Naidoo, K.; Grant, A.D. A Qualitative Study of Patient Motivation to Adhere to Combination Antiretroviral Therapy in South Africa. AIDS Patient Care STDs 2015, 29, 299-306. [CrossRef] [PubMed]

66. Hayden, C.; Neame, R.; Tarrant, C. Patients' adherence-related beliefs about methotrexate: A qualitative study of the role of written patient information. BMJ Open 2015, 5. [CrossRef] [PubMed]

67. McKillop, G.; Joy, J. Patients' experience and perceptions of polypharmacy in chronic kidney disease and its impact on adherent behaviour. J. Ren. Care 2013, 39, 200-207. [CrossRef] [PubMed]

68. Kobue, B.; Moch, S.; Watermeyer, J. "it's so hard taking pills when you don't know what they're for": A qualitative study of patients' medicine taking behaviours and conceptualisation of medicines in the context of rheumatoid arthritis. BMC Health Serv. Res. 2017, 17. [CrossRef]

69. Jeragh-Alhaddad, F.B.; Waheedi, M.; Barber, N.D.; Brock, T.P. Barriers to medication taking among Kuwaiti patients with type 2 diabetes: A qualitative study. Patient Prefer. Adherence 2015, 9, 1491-1503. [CrossRef]

70. Souter, C.; Kinnear, A.; Kinnear, M.; Mead, G. Optimisation of secondary prevention of stroke: A qualitative study of stroke patients' beliefs, concerns and difficulties with their medicines. Int. J. Pharm. Pract. 2014, 22, 424-432. [CrossRef]

71. Bezabhe, W.M.; Chalmers, L.; Bereznicki, L.R.; Peterson, G.M.; Bimirew, M.A.; Kassie, D.M. Barriers and facilitators of adherence to antiretroviral drug therapy and retention in care among adult HIV-positive patients: A qualitative study from Ethiopia. PLoS ONE 2014, 9. [CrossRef]

72. Saleem, F.; Hassali, M.A.; Shafie, A.A.; Atif, M. Drug attitude and adherence: A qualitative insight of patients with hypertension. J. Young Pharm. 2012, 4, 101-107. [CrossRef] [PubMed]

73. Rifkin, D.E.; Laws, M.B.; Rao, M.; Balakrishnan, V.S.; Sarnak, M.J.; Wilson, I.B. Medication adherence behavior and priorities among older adults with CKD: A semistructured interview study. Am. J. Kidney Dis. 2010, 56, 439-446. [CrossRef] [PubMed]

74. van Geffen, E.C.; Hermsen, J.H.; Heerdink, E.R.; Egberts, A.C.; Verbeek-Heida, P.M.; van Hulten, R. The decision to continue or discontinue treatment: Experiences and beliefs of users of selective serotonin-reuptake inhibitors in the initial months-a qualitative study. Res. Soc. Adm. Pharm. 2011, 7, 134-150. [CrossRef]

75. Alhomoud, F.; Dhillon, S.; Aslanpour, Z.; Smith, F. South Asian and Middle Eastern patients' perspectives on medicine-related problems in the United Kingdom. Int. J. Clin. Pharm. 2015, 37, 607-615. [CrossRef]

76. Dehdari, L.; Dehdari, T. The determinants of anti-diabetic medication adherence based on the experiences of patients with type 2 diabetes. Arch. Public Health 2019, 77. [CrossRef]

77. Alodhaib, G.; Alhusaynan, I.; Mirza, A.; Almogbel, Y. Qualitative Exploration of Barriers to Medication Adherence Among Patients with Uncontrolled Diabetes in Saudi Arabia. Pharmacy 2021, 9, 16. [CrossRef] [PubMed]

78. Lyimo, R.A.; De Bruin, M.; Van Den Boogaard, J.; Hospers, H.J.; Van Der Ven, A.; Mushi, D. Determinants of antiretroviral therapy adherence in northern Tanzania: A comprehensive picture from the patient perspective. BMC Public Health 2012, 12. [CrossRef]

79. Chen, L.C.; Chen, T.C.; Huang, Y.B.; Chang, C.S. Disease acceptance and adherence to imatinib in Taiwanese chronic myeloid leukaemia outpatients. Int. J. Clin. Pharm. 2014, 36, 120-127. [CrossRef] 
80. Goldsmith, L.J.; Kolhatkar, A.; Popowich, D.; Holbrook, A.M.; Morgan, S.G.; Law, M.R. Understanding the patient experience of cost-related non-adherence to prescription medications through typology development and application. Soc. Sci. Med. 2017, 194, 51-59. [CrossRef] [PubMed]

81. Ming, L.C.; Hassali, M.A.; Shafie, A.A.; Awaisu, A.; Hadi, M.A.; Al-Haddad, M. Perspectives of heart failure patients in Malaysia towards medications and disease state management: Findings from a qualitative study. J. Public Health 2011, 19, 569-577. [CrossRef]

82. Peláez, S.; Bacon, S.L.; Lacoste, G.; Lavoie, K.L. How can adherence to asthma medication be enhanced? Triangulation of key asthma stakeholders' perspectives. J. Asthma 2016, 53, 1076-1084. [CrossRef] [PubMed]

83. Jaffray, M.; Cardy, A.H.; Reid, I.C.; Cameron, I.M. Why do patients discontinue antidepressant therapy early? A qualitative study. Eur. J. Gen. Pract. 2014, 20, 167-173. [CrossRef] [PubMed]

84. Richardson, J.C.; Liddle, J.; Mallen, C.D.; Roddy, E.; Hider, S.; Prinjha, S.; Ziebland, S. A joint effort over a period of time: Factors affecting use of urate-lowering therapy for long-Term treatment of gout. BMC Musculoskelet. Disord. 2016, 17. [CrossRef] [PubMed] 